\title{
OpenFermion: The Electronic Structure Package for Quantum Computers
}

Jarrod R. McClean, ${ }^{1, *}$ Kevin J. Sung, ${ }^{2}$ Ian D. Kivlichan,,${ }^{1,3}$ Yudong Cao, ${ }^{4,5}$ Chengyu Dai, ${ }^{6}$ E. Schuyler Fried, ${ }^{4,7}$ Craig Gidney, ${ }^{8}$ Brendan Gimby, ${ }^{2}$ Pranav Gokhale, ${ }^{9}$ Thomas Häner, ${ }^{10}$ Tarini Hardikar, ${ }^{11}$ Vojtěch Havlíček, ${ }^{12}$ Oscar Higgott, ${ }^{13}$ Cupjin Huang, ${ }^{2}$ Josh Izaac, ${ }^{14}$ Zhang Jiang, ${ }^{1,}{ }^{15}$ Xinle Liu, ${ }^{16}$ Sam McArdle, ${ }^{17}$ Matthew Neeley, ${ }^{8}$ Thomas O'Brien, ${ }^{18}$ Bryan O'Gorman, ${ }^{19,}{ }^{15}$ Isil Ozfidan, ${ }^{20}$ Maxwell D. Radin, ${ }^{21}$ Jhonathan Romero, ${ }^{4}$ Nicholas Rubin, ${ }^{7}$ Nicolas P. D. Sawaya, ${ }^{4}$ Kanav Setia, ${ }^{11}$ Sukin Sim,${ }^{4}$ Damian S. Steiger, ${ }^{1,10}$ Mark Steudtner, ${ }^{18,22}$ Qiming Sun, ${ }^{23}$ Wei Sun, ${ }^{16}$ Daochen Wang, ${ }^{24}$ Fang Zhang, ${ }^{2}$ and Ryan Babbush ${ }^{1,}{ }^{\dagger}$

\footnotetext{
${ }^{1}$ Google Inc., Venice, CA 90291
}

${ }^{2}$ Department of Electrical Engineering and Computer Science, University of Michigan, Ann Arbor, MI 48109

${ }^{3}$ Department of Physics, Harvard University, Cambridge, MA 02138

${ }^{4}$ Department of Chemistry and Chemical Biology, Harvard University, Cambridge, MA 02138

${ }^{5}$ Zapata Computing Inc., Cambridge 02138

${ }^{6}$ Department of Physics, University of Michigan, Ann Arbor, MI 48109

${ }^{7}$ Rigetti Computing, Berkeley CA 94710

${ }^{8}$ Google Inc., Santa Barbara, CA 93117

${ }^{9}$ Department of Computer Science, University of Chicago, Chicago, IL 60637

${ }^{10}$ Theoretische Physik, ETH Zurich, 8093 Zurich, Switzerland

${ }^{11}$ Department of Physics, Dartmouth College, Hanover, NH 03755

${ }^{12}$ Department of Computer Science, Oxford University, Oxford OX1 3QD, United Kingdom

${ }^{13}$ Department of Physics and Astronomy, University College London, Gower Street, London WC1E 6BT, United Kingdom

${ }^{14}$ Xanadu, 372 Richmond St $W$, Toronto, M5V 1X6, Canada

${ }^{15}$ QuAIL, NASA Ames Research Center, Moffett Field, CA 94035

${ }^{16}$ Google Inc., Mountain View, CA 94043

${ }^{17}$ Department of Materials, University of Oxford, Parks Road, Oxford OX1 3PH, United Kingdom

${ }^{18}$ Instituut-Lorentz, Universiteit Leiden, 2300 RA Leiden, The Netherlands

${ }^{19}$ BQIC, University of California, Berkeley, CA 94720

${ }^{20} D$-Wave Systems Inc., Burnaby, BC

${ }^{21}$ Materials Department, University of California Santa Barbara, Santa Barbara, CA 93106

${ }^{22}$ QuTech, Delft University of Technology, Lorentzweg 1, 2628 CJ Delft, The Netherlands

${ }^{23}$ Division of Chemistry and Chemical Engineering,

California Institute of Technology, Pasadena, CA 91125

${ }^{24}$ Joint Center for Quantum Information and Computer Science, University of Maryland, College Park, MD 20742

(Dated: February 28, 2019)

\begin{abstract}
Quantum simulation of chemistry and materials is predicted to be an important application for both near-term and fault-tolerant quantum devices. However, at present, developing and studying algorithms for these problems can be difficult due to the prohibitive amount of domain knowledge required in both the area of chemistry and quantum algorithms. To help bridge this gap and open the field to more researchers, we have developed the OpenFermion software package (www.openfermion.org). OpenFermion is an open-source software library written largely in Python under an Apache 2.0 license, aimed at enabling the simulation of fermionic and bosonic models and quantum chemistry problems on quantum hardware. Beginning with an interface to common electronic structure packages, it simplifies the translation between a molecular specification and a quantum circuit for solving or studying the electronic structure problem on a quantum computer, minimizing the amount of domain expertise required to enter the field. The package is designed to be extensible and robust, maintaining high software standards in documentation and testing. This release paper outlines the key motivations behind design choices in OpenFermion and discusses some basic OpenFermion functionality which we believe will aid the community in the development of better quantum algorithms and tools for this exciting area of research.
\end{abstract}

\footnotetext{
* Corresponding author: jarrodmcc@gmail.com

$\dagger$ Corresponding author: ryanbabbush@gmail.com
} 


\section{INTRODUCTION}

Recent strides in the development of hardware for quantum computing demand comparable developments in the applications and software these devices will run. Since the inception of quantum computation, a number of promising application areas have been identified, ranging from factoring [1] and solutions of linear equations [2] to simulation of complex quantum materials. However, while the theory has been well developed for many of these problems, the challenge of compiling efficient algorithms for these devices down to hardware realizable gates remains a formidable one. While this problem is difficult to tackle in full generality, significant progress can be made in particular areas. Here, we focus on what was perhaps the original application for quantum devices, quantum simulation.

Beginning with Feynman in 1982 [3], it was proposed that highly controllable quantum devices, later to become known as quantum computers, would be especially good at the simulation of other quantum systems. This notion was later formalized to show how in particular instances, we expect an exponential speedup in the solution of the Schrödinger equation for chemical systems [4-9]. This opens the possibility of understanding and designing new materials, drugs, and catalysts that were previously untenable. There has also been substantial work applying these methods to other fermionic systems such as the Hubbard model [10-12] and Sachdev-Ye-Kitaev model [13, 14]. Since the initial work in this area, there has been great progress developing new algorithms [15-30], tighter bounds and better implementation strategies [27, 28, 31-37], more desirable Hamiltonian representations [38-50], fault-tolerant resource estimates and layouts [51-53], and proof-of-concept experimental demonstrations [54-57]. Moreover, with mounting experimental evidence, variational and hybrid-quantum classical algorithms [58-69] for these systems have been identified as particularly promising approaches when one has limited resources; some speculate these quantum algorithms may even solve classically intractable instances without error-correction.

However, despite immense progress, much work is left to be done in optimizing algorithms in this area for both near-term and far-future devices. Already this field has seen instances where the difference between naive bounds for algorithms and expected numerics for real systems can differ by many orders of magnitude [19, 34-36, 52]. Unfortunately, developing algorithms in this area can require a prohibitive amount of domain expertise. For example, quantum algorithms experts may find the chemistry literature rife with jargon and unknown approximations while chemists find themselves unfamiliar with the concepts used in quantum information. As has been seen though, both have a crucial role to play in developing algorithms for these emerging devices.

For this reason, we introduce the OpenFermion package, designed to bridge the gap between different domain areas and facilitate the development of explicit quantum simulation algorithms for quantum chemistry. The goal of this project is to enable both quantum algorithm developers and quantum chemists to make contributions to this developing field, minimizing the amount of domain knowledge required to get started, and aiding those with knowledge to perform their work more quickly and easily. OpenFermion is an open-source Apache 2.0 licensed software package to encourage community adoption and contribution. It has a modular design and is maintained with strict documentation and testing standards to ensure robust code and project longevity. Moreover, to maximize usefulness within the field, every effort has been made to design OpenFermion as a modular library which is agnostic with respect to quantum programming language frameworks. Through its plugin system, OpenFermion is able to interface with, and benefit from, any of the frameworks being developed for both more abstract quantum software and hardware specific compilation [70-80].

This technical document introduces the first release of the OpenFermion package and proceeds in the following way. We begin by mapping the standard workflow of a researcher looking to implement an electronic structure problem on a quantum computer, giving examples along this path of how OpenFermion aids the researcher in making this process as painless as possible. We aim to make this exposition accessible to all interested readers, so some background in the problem is provided as well. The discussion then shifts to the core and derived data structures the package is designed around. Following this, some example applications from real research projects are discussed, demonstrating real-world examples of how OpenFermion can streamline the research process in this area. Finally, we finish with a brief discussion of the open source philosophy of the project and plans for future developments.

\section{QUANTUM WORKFLOW PIPELINE}

In this section we step through one of the primary problems in entering quantum chemistry problems to a quantum computer. Specifically, the workflow for translating a problem in quantum chemistry to one in quantum computing. This process begins by specifying the problem of interest, which is typically finding some molecular property for a particular state of a molecule or material. This requires one to specify the molecule of interest, usually through the positions of the atoms and their identities. However, this problem is also steeped in some domain specific terminology, especially with regards to symmetries and choices of discretizations. OpenFermion helps to remove many of these particular difficulties. Once the problem has been specified, several computational intermediates must be computed, 
namely the bare two-electron integrals in the chosen discretization as well as the transformation to a molecular orbital basis that may be needed for certain correlated methods. From there, translation to qubits may be performed by one of several mappings. The goal of this section is to both detail this pipeline, and show at each step how OpenFermion may be used to help perform it with ease.

\section{A. Molecule specification and input generation}

Electronic structure typically refers to the problem of determining the electronic configuration for a fixed set of nuclear positions assuming non-relativistic energy scales. To begin with, we show how a molecule is defined within OpenFermion, then continue on to describe what each component represents.

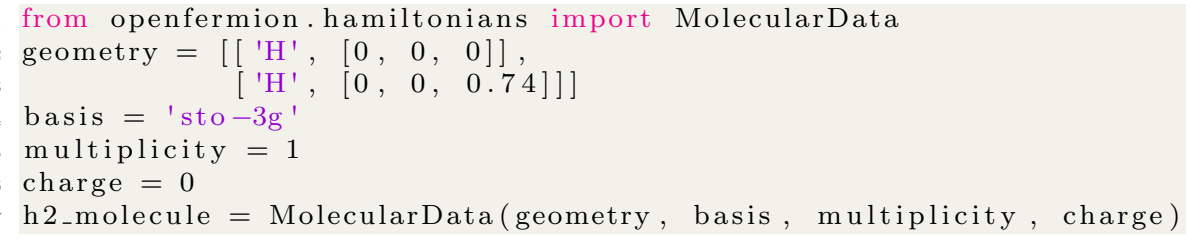

Listing 1. Defining a simple $\mathrm{H}_{2}$ instance in OpenFermion.

The specification of a molecular geometry implicitly assumes the Born-Oppenheimer approximation, which treats the nuclei as fixed point charges, and the ground state electronic energy is a parametric function of their positions. When the positions of the nuclei are specified, the electronic structure problem can be restated as finding the eigenstates of the Hamiltonian operator

$$
H(\mathbf{r} ; \mathbf{R})=-\sum_{i} \frac{\nabla_{r_{i}}^{2}}{2}-\sum_{i, j} \frac{Z_{j}}{\left|R_{j}-r_{i}\right|}+\sum_{i<j} \frac{1}{\left|r_{i}-r_{j}\right|}+\sum_{i<j} \frac{Z_{i} Z_{j}}{\left|R_{i}-R_{j}\right|}
$$

where we have used atomic units (i.e. $\hbar=1$ ), $r_{i}$ represent the positions of electrons, $R_{i}$ represent the positions of nuclei, and $Z_{i}$ are the charges of nuclei. In our example from OpenFermion, we see that the nuclear positions may be specified by a set of $x, y, z$ coordinates along with atom labels as

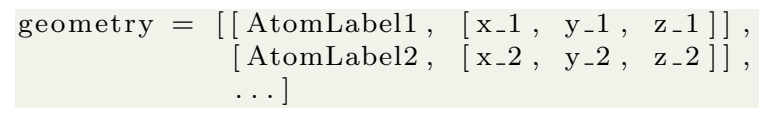

A reasonable set of nuclear positions $R$ for a given molecule can often be given from experimental data, empirical models, or a geometry optimization. A geometry optimization minimizes the lowest eigenvalue of the above Hamiltonian as a function of the nuclear position, to some local, stable optimum. These nuclear configurations are sometimes called equilibrium structures with respect to the level of theory used to calculate them.

As we focus on the non-relativistic Hamiltonian, there is no explicit dependence on electron spin. As a result, the total electronic spin and one of its components (canonically chosen to be the $Z$ direction) form good quantum numbers for the Hamiltonian. That is, the Hamiltonian can be made block diagonal with separate spin labels for each block. Explicitly including this symmetry offers a number of computational advantages, including smaller spaces to explore, as well as the ability to access excited states that are the lowest energy state within a particular spin manifold using ground state methods [81]. In particular, we parameterize these manifolds by the spin multiplicity defined by

$$
\text { multiplicity }=2 S+1
$$

where the eigenstates of the $S^{2}$ operator have, by definition, eigenvalues of $S(S+1)$. In our example, we have used a singlet state with $S=0$ to specify we are looking for the lowest singlet energy state. This was specified simply by

multiplicity $=1$

and we note that a multiplicity of 3 (a triplet state) might have also been of interest for this particular system.

Given a particular set of nuclei, it is often the case that a system with the same number of electrons as protons (or electronically neutral system) is the most stable. However, sometimes one wishes to study systems with a non-neutral charge such as the +1 cation or -1 anion of the system. In that case, one may specify the charge, which is defined to be the number of electrons in the neutral system minus the number of electrons in the system of interest. In our example, we specified the neutral hydrogen atom, with

charge $=0$ 
Finally, to specify the computational problem to be solved, rather than simply the molecule itself, it is necessary to define the basis set. This is analogous to, in some loose sense, how one might define a grid to solve a a simple partial differential equation such as the heat-equation. In chemistry, much thought has gone into optimizing specialized basis sets to pack the essential physics of the problems into functions that balance cost and accuracy. A list of some of the most common basis sets expressed as sums of Gaussians can be found in the EMSL basis set exchange [82]. In this case, we specify the so-called minimal basis set "sto-3g", which stands for 3 Gaussians (3G) used to approximate Slater-type orbitals (STO). This is done through the line

basis $=$ 'sto $-3 \mathrm{~g}$ '

In future implementations, the code may additionally support parametric or user defined basis sets with similar syntax. At present, the code supports basis sets that are implemented within common molecular electronic structure packages that will be described in more detail in the next section. With the above specifications, the chemical problem is now well defined; however, several steps remain in mapping to a qubit representation.

\section{B. Integral generation}

After the specification of the molecule as in the previous section, it becomes necessary to do some of the numerical work in generating the problem to be solved. In OpenFermion we accomplish this through plugin libraries that interface with existing electronic structure codes. At present there are supported interfaces to Psi4 [83] and PySCF [84], with plans to expand to other common packages in the future. One needs to install these plugin libraries separately from the core OpenFermion library (instructions are provided at www.openfermion.org that includes a Docker alternative installing all these packages). We made the decision to support these packages as plugins rather than directly integrating them for several reasons. First, some of these packages have different open source licenses than OpenFermion. Second, these packages may require more intricate installations and do not run on all operating systems. Finally, in the future one may wish to use OpenFermion in conjunction with other electronic structure packages which might support methods not implemented in Psi4 and PySCF. The plugin model ensures the modularity necessary to maintain such compatibilities as the code evolves.

Once one has chosen a particular basis and enforced the physical anti-symmetry of electrons, the electronic structure problem may be written exactly in the form of a second quantized electronic Hamiltonian as

$$
H(R)=\sum_{i j} h_{i j}(R) a_{i}^{\dagger} a_{j}+\frac{1}{2} \sum_{i j k l} h_{i j k l}(R) a_{i}^{\dagger} a_{j}^{\dagger} a_{k} a_{l} .
$$

The coefficients $h_{i j}$ and $h_{i j k l}$ are defined by the basis set that was chosen for the problem (sto-3g in our example); however the computation of these coefficients in general can be quite involved. In particular, in many cases it makes sense to perform a Hartree-Fock calculation first and transform the above integrals into the molecular orbital basis that results. This has the advantage of making the mean-field state easy to represent on both a classical and quantum computer, but introduces some challenges with regards to cost of the integral transformation and convergence of the Hartree-Fock calculation for challenging systems. For example, it is necessary to specify an initial guess for the orbitals of Hartree-Fock, the method used to solve the equations, and a number of other numerical parameters that affect convergence.

In OpenFermion we address this problem by choosing a reasonable set of default parameters and interfacing with well developed electronic structure packages through a set of plugin libraries to supply the information desired. For example, using the OpenFermion-Psi4 plugin, one can obtain the two-electron integrals $h_{i j k l}$ for this molecule in the MO basis in the Psi4 electronic structure code by simply executing

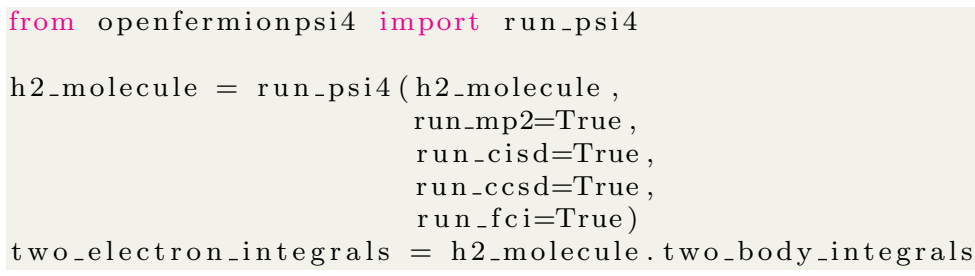

where the h2_molecule is that defined in the previous section and when run in this way, one may easily access the MP2, CISD, CCSD, and FCI energies. This will return the computed two-electron integrals in the Hartree-Fock molecular orbital basis. Moreover, one has direct access to other common properties such as the molecular orbital coefficients through simple commands such as

orbitals $=$ h2_molecule.canonical_orbitals 
One may also read the 1- and 2-electron reduced density matrices from CISD and FCI and the converged coupled cluster amplitudes from CCSD. These values are all conveniently stored to disk using an HDF5 interface that only loads the properties of interest from disk for convenient analysis of data after the fact.

The plugins are designed to ideally function uniformly without exposing the details of the underlying package to the user. For example, the same computation may be accomplished using PySCF through the OpenFermion-PySCF plugin by executing the commands

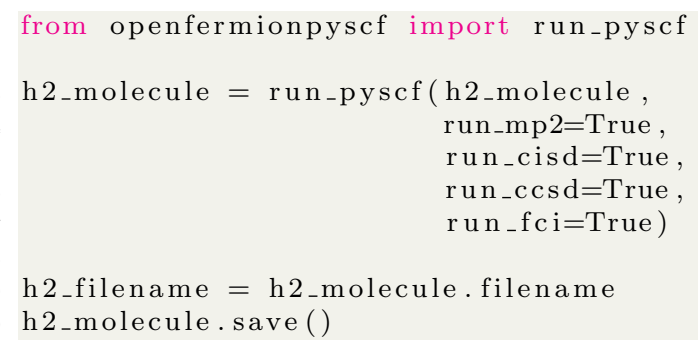

This allows the user to prepare a data structure representing the molecule that is agnostic to the package with which it was generated. In the future, additional plugins will support a wider range of electronic structure packages to meet the growing demands of users. In the last line of this example, we introduce the save feature of the MolecularData class. We note that this is called by default by the plugins generating the data, but introduce it here to emphasize the fact that this data is conveniently stored for future use. This allows one to retrieve any data about this molecule in the future without an additional calculation through

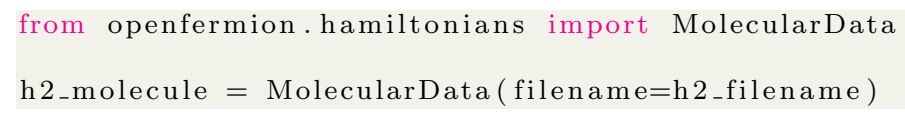

where h2_filename is the filename one chose to store the $\mathrm{H}_{2}$ data under. By using decorated Python attributes and HDF5 storage, the loading of data in this way is done on-demand for any array-like object such as integrals. That is, loading the molecule in this way has a minimal memory footprint, and large quantities such as density matrices or integrals are only loaded when the attribute is accessed, for example

one_body_integrals $=$ h2_molecule.one_body_integrals

and is performed seamlessly in the background such that no syntax is required beyond accessing the attribute.

\section{Mapping to qubits}

After the problem has been recast in the second quantized representation, it remains to map the problems to qubits. Electrons are anti-symmetric indistinguishable particles, while qubits are distinguishable particles, so some care must be taken in mapping between the two. There are now many maps that respect the correct particle statistics, and several of the most common are currently implemented within OpenFermion. In particular, the Jordan-Wigner (JW) [85], Bravyi-Kitaev (BK) [38, 86], and Bravyi-Kitaev super fast (BKSF) [47] transformations are currently supported in OpenFermion. Each of these has different properties with regard to the Hamiltonians that are produced, which may offer benefits to different types of algorithms or experiments. OpenFermion attempts to remain agnostic to the particular transformation preferred by the user.

To give a concrete example, the above Hamiltonian may be mapped to a qubit Hamiltonian through the use of the Jordan-Wigner transformation by

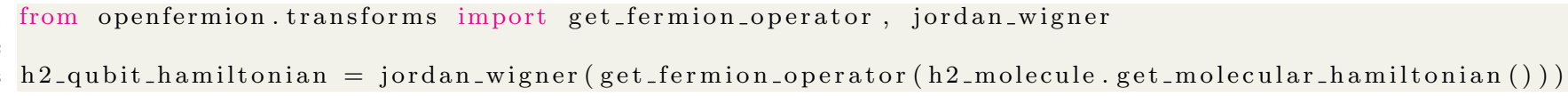

which returns a qubit operator representing the Hamiltonian after the Jordan-Wigner transformation. This data structure will be explored in more detail later in this paper, but it provides the complete specification of the Hamiltonian acting on qubits in a convenient format.

Note that, in addition to mapping fermionic Hamiltonians to qubit operators, OpenFermion also supports mapping bosonic Hamiltonians - those describing systems of indistinguishable symmetric particles — to propagation modes of light, or qumodes, through the use of plugins to quantum optics-based simulators. In future iterations of OpenFermion, algorithms mapping bosonic systems to qubits may additionally be implemented. 


\section{Numerical testing}

While the core functionality of OpenFermion is designed to provide a map between the space of electronic structure problems and qubit-based quantum computers, some functionality is provided for numerical simulation. This can be helpful for debugging or prototyping new algorithms. For example, one could check the spectrum of the above qubit Hamiltonian after the Jordan-Wigner transformation by performing

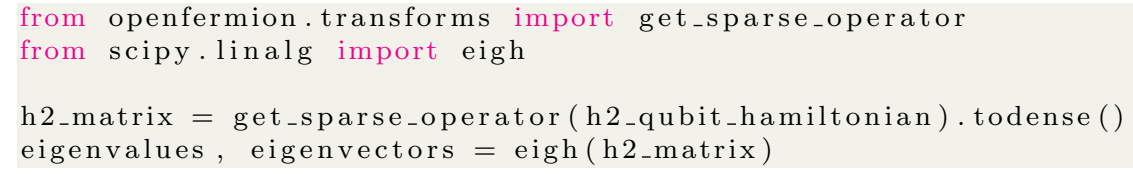

which yields the exact eigenvalues and eigenvectors of the $\mathrm{H}_{2}$ Hamiltonian in the computational basis.

\section{E. Compiling circuits for quantum algorithms}

The core of OpenFermion consists of tools for obtaining and manipulating representations of quantum operators. These tools and representations are useful for compiling quantum circuits, but the best way to perform this compilation depends on the particular algorithm and hardware used. Many groups have their own software for performing this compilation. OpenFermion is intended to be agnostic with respect to the particular hardware and compilation platform; we delegate the final compilation step to platform-specific plugins. At the time of writing, plugins are supported for the Cirq, Forest, and Strawberry Fields [80] frameworks. These plugins are called OpenFermion-Cirq, Forest-OpenFermion, and SFOpenBoson, respectively. Below, we provide examples of using these plugins to compile quantum circuits.

\section{OpenFermion-Cirq Example}

In our first example, we assume the user has a hydrogen molecule object stored in the variable h2_molecule and use Cirq and OpenFermion-Cirq to construct a circuit that simulates time evolution by the molecular Hamiltonian via a product formula implemented with the "low rank" strategy described in [49]. We'll compile just one Trotter step and perform a circuit optimization which merges single-qubit rotations before displaying the circuit.

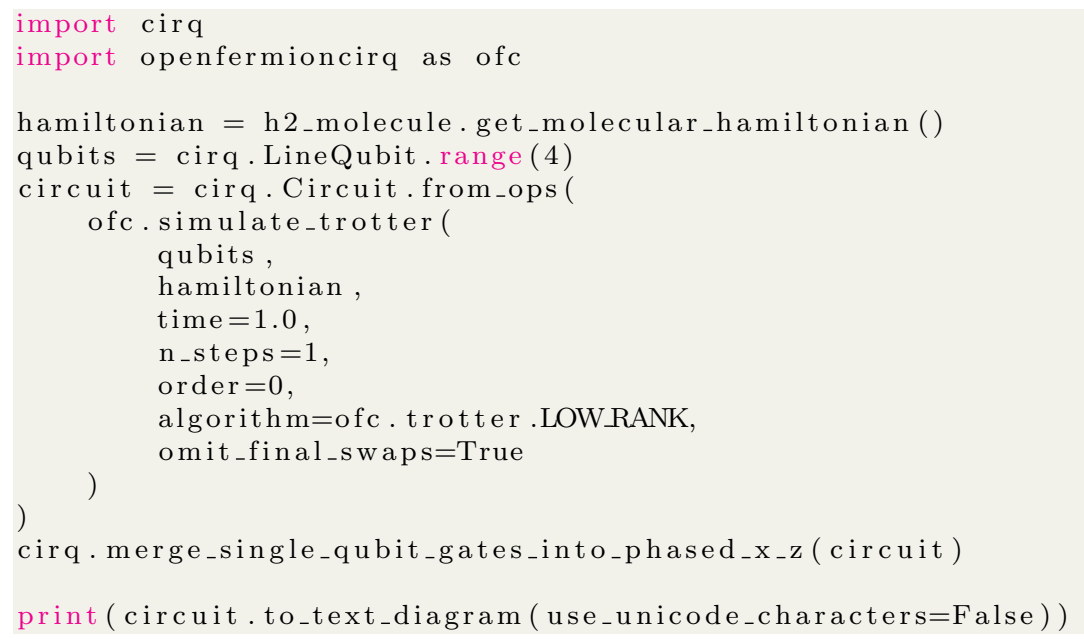

Executing this code produces an ASCII circuit diagram which we truncate and display below:

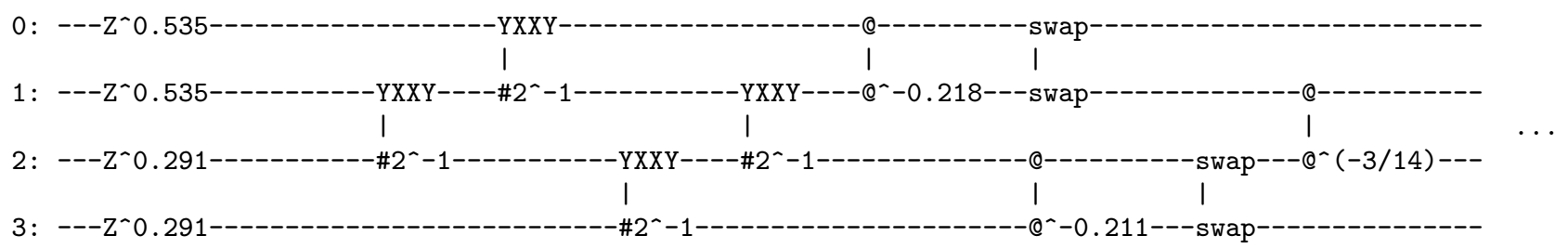


In our next example, we construct a circuit which prepares the ground state of the tunneling term of a FermiHubbard model Hamiltonian. The Fermi-Hubbard model has the Hamiltonian

$$
-t \sum_{\langle i, j\rangle, \sigma}\left(a_{i, \sigma}^{\dagger} a_{j, \sigma}+a_{j, \sigma}^{\dagger} a_{i, \sigma}\right)+U \sum_{i} a_{i, \uparrow}^{\dagger} a_{i, \uparrow} a_{i, \downarrow}^{\dagger} a_{i, \downarrow}
$$

which is composed of a tunneling term (on the left) and an interaction term (on the right). The tunneling term is a quadratic Hamiltonian and its ground state is a Slater determinant. More generally, eigenstates of quadratic Hamiltonians are known as fermionic Gaussian states.

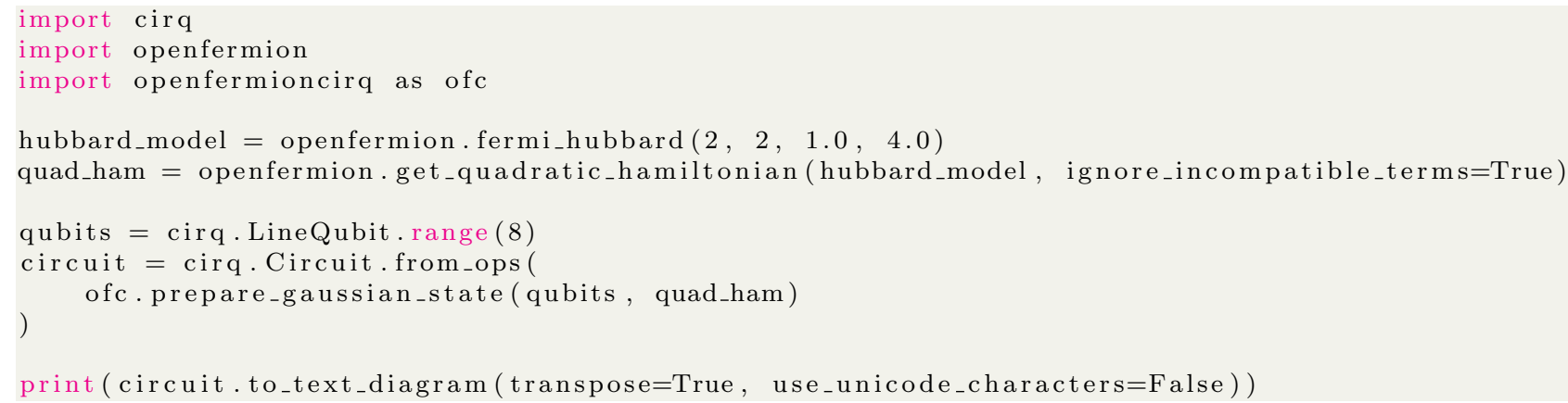

Executing this code produces the following ASCII circuit diagram:

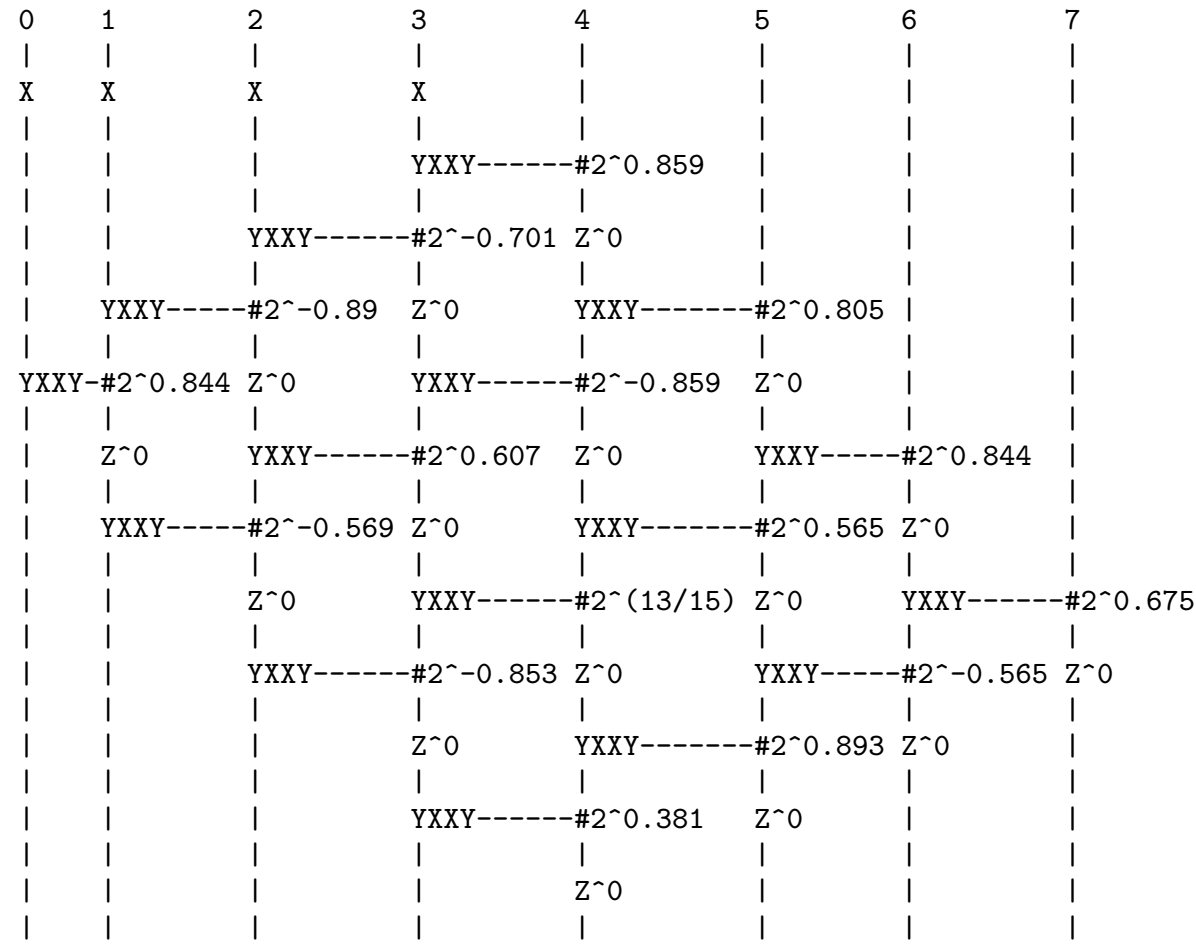

\section{Forest-OpenFermion Example}

In this section we describe the interface between OpenFermion and Rigetti's quantum simulation environment called Forest. The interface provides a method of transforming data generated in OpenFermion to a similar representation in pyQuil. For this example we use OpenFermion to build a four-site single-band periodic boundary Hubbard model and apply first-order Trotter time-evolution to a starting state of two localized electrons of opposite spin.

The Forest-OpenFermion plugin provides the routines to inter-convert between the OpenFermion QubitOperator data structure and the synonymous data structure in pyQuil called a PauliSum.

from openfermion.ops import QubitOperator

from forestopenfermion import pyquilpauli_to_qubitop, qubitop_to_pyquilpauli 
The FermionOperator in OpenFermion can be used to translate the mathematical expression of the Hamiltonian directly to executable code. While we show how this model can be built in one line using the OpenFermion hamiltonians module below, here we take the opportunity to demonstrate the ease of creating such models for study that could be easily modified as desired. Given the Hamiltonian of the Hubbard system

$$
H=-1 \sum_{\langle i, j\rangle, \sigma}\left(a_{i, \sigma}^{\dagger} a_{j, \sigma}+a_{j, \sigma}^{\dagger} a_{i}\right)+4 \sum_{i=0}^{3} a_{i, \alpha}^{\dagger} a_{i, \alpha} a_{i, \beta}^{\dagger} a_{i, \beta}
$$

where $\langle\cdot, \cdot\rangle$ indicates nearest-neighbor spatial lattice positions and $\sigma$ takes on values of $\alpha$ and $\beta$ signifying spin-up or spin-down, respectively, the code to build this Hamiltonian is as follows:

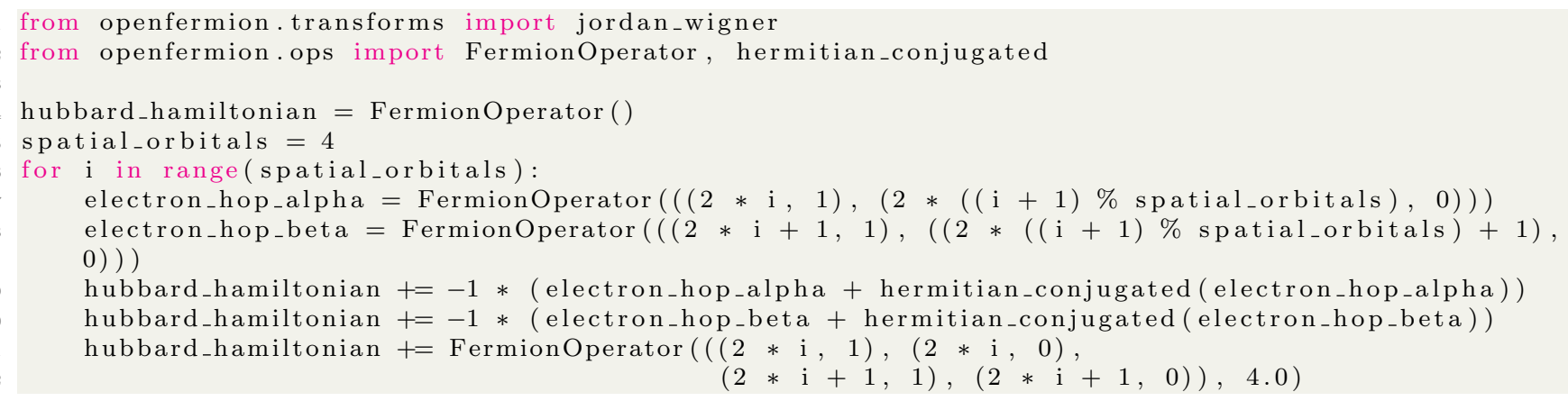

In the above code we have implicitly used even indexes [0,2,4,6] as $\alpha$ spin-orbitals and odd indexes [1,3,5,7] as $\beta$ spin-orbitals. The same model can be built using the OpenFermion Hubbard model builder routine in the hamiltonians module with a single function call.

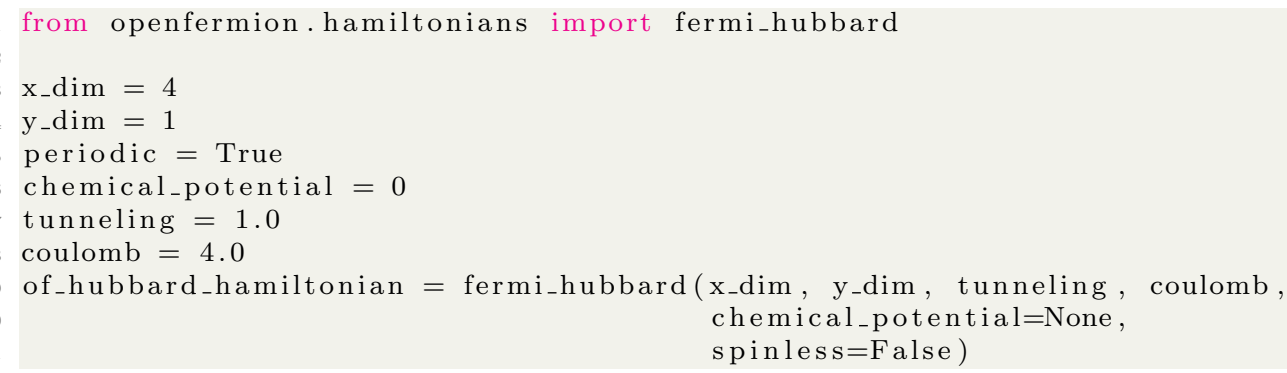

Using the Jordan-Wigner transform functionality of OpenFermion, the Hubbard Hamiltonian can be transformed to a sum of QubitOperators which are then transformed to pyQuil PauliSum objects using routines in the ForestOpenFermion plugin imported earlier.

hubbard_term_generator = jordan_wigner(hubbard_hamiltonian)

pyquil_hubbard_generator = qubitop_to_pyquilpauli(hubbard_term_generator)

With the data successfully transformed to a pyQuil representation, the pyQuil exponentiate routine is used to generate a circuit corresponding to first-order Trotter evolution for $t=0.1$.

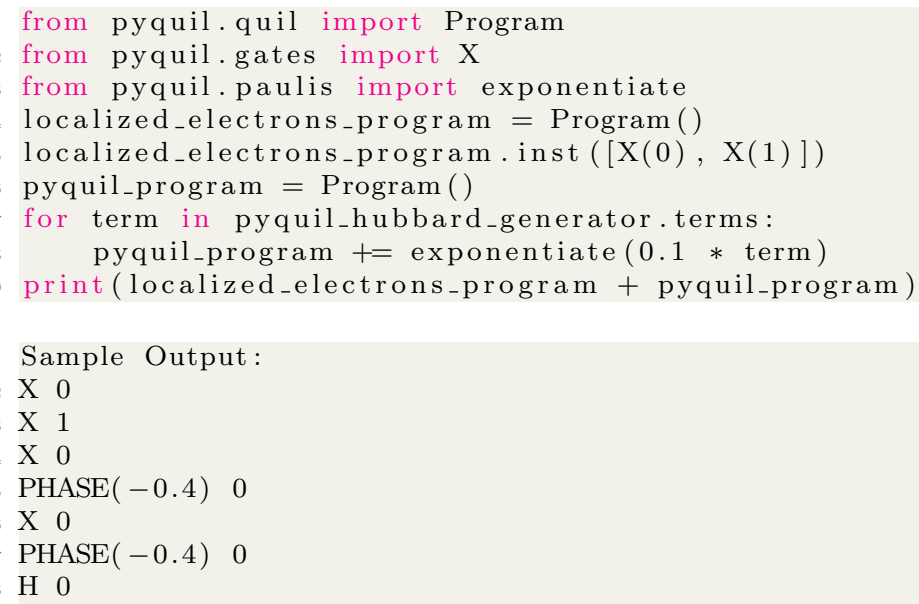




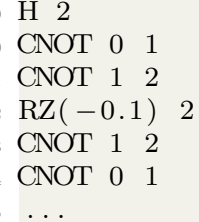

The output is the first few lines of the Quil [72] program that sets up the two-localized electrons on the first spatial site and then applies the time-propagation circuit. This Quil program can be sent to the Forest cloud API for simulation or for execution on hardware.

\section{SFOpenBoson Example}

Quantum optics and continuous-variable (CV) quantum computation provide a well-suited environment for the quantum simulation of bosons. In this example, we describe the interface between OpenFermion and Xanadu's quantum optics-based simulation environment Strawberry Fields, and simulate a Bose-Hubbard model using the CV gate set and propagation modes of light.

Consider the following Bose-Hubbard Hamiltonian, describing bosons on a lattice with on-site interaction strength $U=1.5$ and chemical potential $\mu=0.5$ :

$$
H=-\sum_{\langle i, j\rangle} b_{i}^{\dagger} b_{j+1}+\frac{1.5}{2} \sum_{k=1}^{N-1} b_{k}^{\dagger} b_{k}\left(b_{k}^{\dagger} b_{k}-1\right)-0.5 \sum_{k=1}^{N} b_{k}^{\dagger} b_{k} .
$$

Here, $b_{i}^{\dagger}$ and $b_{i}$ are the bosonic creation and annhilation operators respectively acting on qumode $i$. Using OpenFermion, it is a one line procedure to generate this Hamiltonian for a $2 \times 2$ lattice:

from openfermion. hamiltonians import bose_hubbard

$\mathrm{H}=$ bose_hubbard $(2,2$, tunneling $=1$, interaction $=1.5$, chemical_potential $=0.5)$

We can now use the SFOpenBoson plugin to simulate this Hamiltonian in Strawberry Fields. The SFOpenBoson plugin provides operations to convert the OpenFermion BosonOperator/QuadOperator data structure to the standard operations and gates used in continuous-variable quantum computation. Using the Hamiltonian defined above, we can do this as follows:

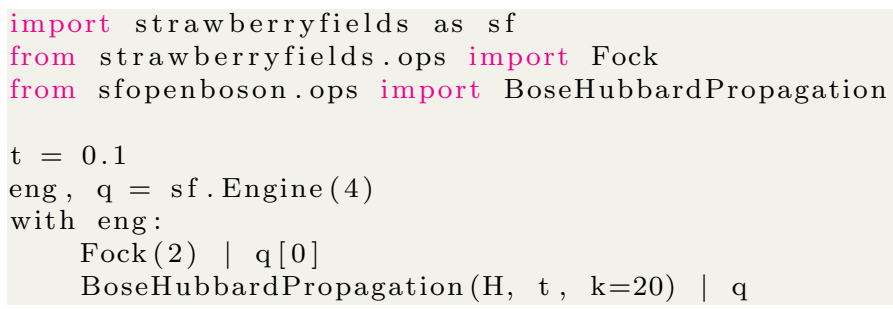

In the above code, we create a two qumode quantum circuit with the system initialized in Fock state $|2,0,0,0\rangle$, and then perform the time evolution for $t=0.1$ using the Lie product formula truncated to 20 terms. Finally, we run the circuit simulation, and print the applied quantum gates:

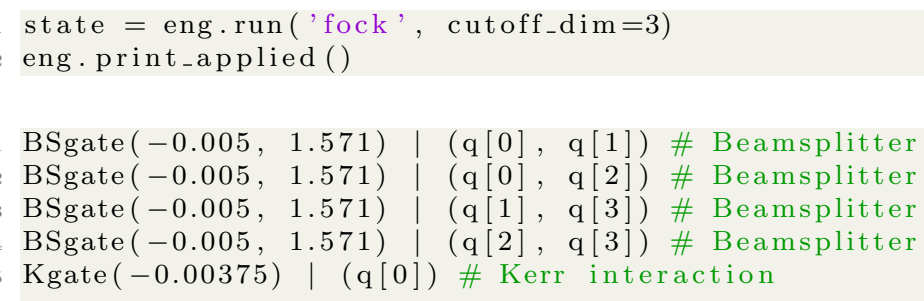

The output above lists the first few gates applied, and the corresponding qumodes they act on, for the Bose-Hubbard simulation. 


\section{DATA STRUCTURES}

In this section we describe some of the data structures defined by OpenFermion. We begin with the most general data structures: FermionOperator, QubitOperator, and BosonOperator. These represent arbitrary linear combinations of operators from the chosen basis; for instance, for FermionOperator, the basis is that of fermionic creation and annihilation operators. Later on we describe data structures such as InteractionOperator which represent operators with additional structure that warrants storage in a different format.

\section{A. FermionOperator data structure}

In the above examples, we saw that an intermediate representation for the molecular problems were objects known as FermionOperators. Fermionic systems are often treated in second quantization, where anti-symmetry requirements are stored in the operators rather than in explicit wavefunction anti-symmetrization and arbitrary operators can be expressed using fermionic creation and annihilation operators $a_{p}^{\dagger}$ and $a_{q}$. Supposing that $p=1, q=0$, such operators could be represented within OpenFermion simply as

from openfermion.ops import FermionOperator

$\mathrm{a}_{-} \mathrm{p}_{-}$dagger $=$FermionOperator $\left({ }^{\prime} 1 \wedge\right.$ ' $)$

$\mathrm{a}_{-} \mathrm{q}=$ FermionOperator $\left({ }^{\prime} 0^{\prime}\right)$

These operators enforce fermionic statistics in the system by satisfying the fermionic anti-commutation relations,

$$
\left\{a_{p}^{\dagger}, a_{q}^{\dagger}\right\}=\left\{a_{p}, a_{q}\right\}=0 \quad\left\{a_{p}^{\dagger}, a_{q}\right\}=\delta_{p q} .
$$

where $\{A, B\} \equiv A B+B A$. The raising operators $a_{p}^{\dagger}$ act on the fermionic vacuum state, $|v a c\rangle$, to create fermions in spin-orbitals, which are single-particle spatial density functions. The connection to first quantization and explicit anti-symmetrization in Slater determinants can be seen if electron $j$ is represented in a space of spin-orbitals $\left\{\phi_{p}\left(r_{j}\right)\right\}$. Then $a_{p}^{\dagger}$ and $a_{p}$ populate fermions in Slater determinants through the equivalence,

$$
\left\langle r_{0}, \ldots, r_{\eta-1}\left|a_{p_{0}}^{\dagger} \cdots a_{p_{\eta-1}}^{\dagger}\right| v a c\right\rangle=\sqrt{\frac{1}{\eta !}}\left|\begin{array}{cccc}
\phi_{p_{0}}\left(r_{0}\right) & \phi_{p_{1}}\left(r_{0}\right) & \cdots & \phi_{p_{\eta-1}}\left(r_{0}\right) \\
\phi_{p_{0}}\left(r_{1}\right) & \phi_{p_{1}}\left(r_{1}\right) & \cdots & \phi_{p_{\eta-1}}\left(r_{1}\right) \\
\vdots & \vdots & \ddots & \vdots \\
\phi_{p_{0}}\left(r_{\eta-1}\right) & \phi_{p_{1}}\left(r_{\eta-1}\right) & \cdots & \phi_{p_{\eta-1}}\left(r_{\eta-1}\right)
\end{array}\right|
$$

which instantiates a system of $\eta$ fermions.

Arbitrary fermionic operators on the space of $N$ spin-orbitals can be represented by weighted sums of products of these raising and lowering operators. The following is an example of one such "fermion operator",

$$
W=(1+2 i) a_{4}^{\dagger} a_{3} a_{9} a_{3}^{\dagger}-4 a_{2}=-(1+2 i) a_{4}^{\dagger} a_{3}^{\dagger} a_{9} a_{3}-(1+2 i) a_{4}^{\dagger} a_{9}-4 a_{2} .
$$

In the second equality above we have used the anti-commutation relations of Eq. (6) to reorder the ladder operators in $W$ into a unique "normal-ordered" form, defined so that raising operators always come first and operators are ordered in descending order of the fermionic mode on which they act. These rules are all handled transparently within OpenFermion so that essential physics are not violated. For example, the $W$ operator could be defined within OpenFermion as

from openfermion.ops import FermionOperator

$\mathrm{W}=(1+2 \mathrm{j}) *$ FermionOperator $\left({ }^{\prime} 4^{\wedge} 393^{\wedge}{ }^{\prime}\right)-4 *$ FermionOperator $\left(' 2^{\prime}\right)$

and the "normal-ordering" can be simply performed by

from openfermion. utils import normal_ordered

W_normal_ordered = normal_ordered $(\mathrm{W})$

So long as ladder operators are manipulated in a fashion that is consistent with Eq. (6), addition, multiplication, and integer exponentiation are well defined for fermion operators. For instance, $W^{4}$ and $W / 82-3 W^{2}$ are also examples of fermion operators, and are readily available in OpenFermion through standard arithmetic manipulations of the operators. For example

$\mathrm{W} \_4=\mathrm{W} * * 4$

print(normal_ordered (W_4)) 
where in the second line, we find if we further use the normal ordered function on this seemingly complicated object, that it in fact evaluates to zero.

Internally, the FermionOperator data structure uses a hash table (currently implemented using a Python dictionary). The keys of the dictionary encode a sequence of raising and lowering operators and the value of that entry stores the coefficient. The current implementation of this class encodes the sequence of ladder operators using a tuple of 2-tuples where the 2-tuples represent ladder operators. The first element of each 2-tuple is an int specifying which fermionic mode the ladder operator acts on and the second element of each 2-tuple is a Boolean specifying whether the operator is raising (True) or lowering (False); thus, the encoding of the ladders operators can be expressed as

$$
\left(p \in \mathbb{N}_{0}, \gamma \in \mathbb{Z}_{2}\right) \mapsto\left\{\begin{array}{ll}
a_{p}^{\dagger} & \gamma=1 \\
a_{p} & \gamma=0
\end{array} .\right.
$$

The sequence of ladder operators is thus specified by a sequence of the 2-tuples just defined. Some examples of the ladder operator sequence encodings are shown below,

$$
() \mapsto \mathbb{1} \quad((2,0),) \mapsto a_{2} \quad((4,1),(9,0)) \mapsto a_{4}^{\dagger} a_{9} \quad((4,1),(3,0),(9,0),(3,1)) \mapsto a_{4}^{\dagger} a_{3} a_{9} a_{3}^{\dagger} .
$$

which can also be used to initialize a FermionOperator as a user as

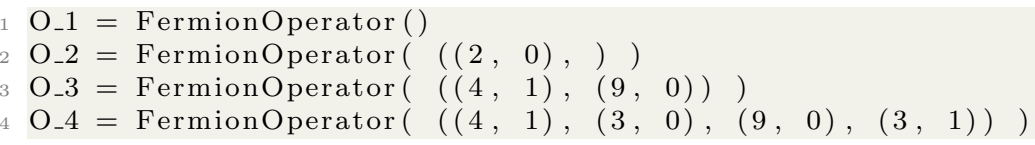

While this is the internal representation of sequences of ladder operators in the FermionOperator data structure, OpenFermion also supports a string representation of ladder operators that is more human-readable. One can initialize FermionOperators using the string representation and when one calls print() on a FermionOperator, the operator is printed out using the string representation. The carat symbol "^ " represents raising, and its absence implies the lowering operator. Below are some self-explanatory examples of our string representation,

$$
\prime^{\prime} \mapsto \mapsto \mathbb{1} \quad ' 2^{\prime} \mapsto a_{2} \quad 4^{\wedge} 9^{\prime} \mapsto a_{4}^{\dagger} a_{9} \quad{ }^{\prime} 4^{\wedge} 393^{\wedge}{ }^{\prime} \mapsto a_{4}^{\dagger} a_{3} a_{9} a_{3}^{\dagger} .
$$

that translate to code as

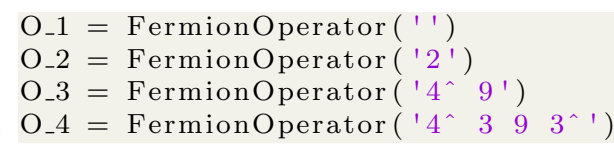

A hash table data structure was chosen to facilitate efficient combination of large FermionOperators through arithmetic operations. This is preferred over an unstructured array of terms due to its native implementation in Python as well as the fact that duplicate terms are nearly automatically combined at a cost that is constant time for modestly sized examples. Similar scaling could be achieved through other data structures, but at increased complexity of implementation in the Python ecosystem. As motivation for this choice, we include in the example section two important use cases of the FermionOperator: the computation of Trotter error operators and the symbolic Fourier transformation.

\section{B. QubitOperator data structure}

Continuing from the example above, once the intermediate FermionOperators have been produced, they must be mapped to the language of quantum computers, or qubits. This is handled within OpenFermion through the QubitOperator data structure. Fundamentally this operator structure is based off the Pauli spin operators and the identity, defined by

$$
I=\left(\begin{array}{ll}
1 & 0 \\
0 & 1
\end{array}\right) \quad X=\left(\begin{array}{cc}
0 & 1 \\
1 & 0
\end{array}\right) \quad Y=\left(\begin{array}{cc}
0 & -i \\
i & 0
\end{array}\right) \quad Z=\left(\begin{array}{cc}
1 & 0 \\
0 & -1
\end{array}\right) .
$$

Tensor products of Pauli operators form a basis for the space of Hermitian operators; thus, it is possible to express any Hermitian operator of interest using just these few operators and their products. If one indexes the qubit a particular operator acts on, such as $X_{i}$, that defines action by $X$ on the $i^{\text {th }}$ qubit (and implicitly action by $I$ on all others). In OpenFermion one may wish to express an operator such as

$$
O=Z_{1} Z_{2}+X_{1}+X_{2}
$$

that could be initialized as 
from openfermion.ops import QubitOperator

$\mathrm{O}=$ QubitOperator ('Z1 Z2') + QubitOperator('X1') + QubitOperator('X2')

Similar to FermionOperator, QubitOperator is implemented internally using a hash table data structure through the native Python dictionary. This choice allows a good level of base efficiency for most arithmetic operators while harnessing the features of the Python dictionary to ease implementation details. The keys used in this implementation are similarly tuples of tuples that define the type of operator and the qubit it acts on,

$$
\left(O \in\{X, Y, Z\}, i \in \mathbb{N}_{0}\right) \mapsto O_{i}
$$

This internal representation may be used to initialize QubitOperators such as the operator $X_{1} X_{2}$ as

$\mathrm{O}=$ QubitOperator $\left(\left(\left(1, \mathrm{X}^{\prime}\right),\left(2, \mathrm{X}^{\prime}\right)\right)\right)$

or alternatively as seen above, a convenient string initializer is also available, which for the same operator could be used as

$\mathrm{O}=$ QubitOperator ('X1 X2')

\section{BosonOperator and QuadOperator data structures}

In addition to the FermionOperator, OpenFermion also provides preliminary support for bosonic systems through the BosonOperator data structure. Like with fermionic systems, bosonic systems are commonly treated in second quantization, using the symmetric bosonic creation and annihilation operators $b_{q}^{\dagger}$ and $b_{q}$. These enforce bosonic statistics by satisfying the canonical bosonic commutation relations,

$$
\left[a_{p}^{\dagger}, a_{q}^{\dagger}\right]=\left[a_{p}, a_{q}\right]=0, \quad\left[a_{p}^{\dagger}, a_{q}\right]=\delta_{p q}
$$

and, analogously to the fermionic case, act on the vacuum state to create/annihilate bosons in the Fock space:

$$
a_{p}^{\dagger n}|v a c\rangle=|\underbrace{0,0, \ldots, 0}_{p-1}, n, 0, \ldots, 0\rangle .
$$

Due to these similarities, the BosonOperator is inherited from the same SymbolicOperator class as the FermionOperator, and thus retains many of the same properties, including the internal representation through the use of a hash table, and forms of input (terms can be specified as a 2-tuple or in the more human-readable string representation). For example, constructing the operator $b_{3}^{\dagger} b_{5} b_{1}^{\dagger} b_{4}$ can be done by the user as

from openfermion.ops import BosonOperator

$\mathrm{O}=$ BosonOperator (' $\left.3{ }^{\wedge} 51^{\wedge} 4^{\prime}\right)$

Note that, unlike fermionic ladder operators, bosonic ladder operators acting on different subsystems commute past each other. Therefore, the terms of this operator will be stored as " $1 \wedge 3^{\wedge} 45$ ", with indices ordered in ascending order from left to right. To recover the unique "normal-ordered" form, with raising operators always to the left of lowering operators, the user can use the normal ordered function provided to automatically rearrange the operator terms as per the commutation relations:

from openfermion.utils import normal_ordered

normal_ordered (BosonOperator ('0 $0^{\wedge}$ ') )

which returns " 1.0[]$+1.0\left[0^{\wedge} 0\right] "$, corresponding to $b_{0} b_{0}^{\dagger}=I+b_{0}^{\dagger} b_{0}$.

In addition to bosonic raising and lowering operators, it is common when studying bosonic systems to also consider dynamics in the phase space $(q, p)$. The Hermitian position and momentum operators $q_{i}$ and $p_{i}$ act upon this phase space, and are defined in terms of the bosonic ladder operators,

$$
q_{i}=\sqrt{\frac{\hbar}{2}}\left(b_{i}+b_{i}^{\dagger}\right), \quad p_{i}=-i \sqrt{\frac{\hbar}{2}}\left(b_{i}-b_{i}^{\dagger}\right) .
$$

These operators are Hermitian, and are referred to as the quadrature operators. They satisfy the commutation relation $\left[q_{i}, p_{j}\right]=i \hbar \delta_{i j}$, where $\hbar$ depends on convention, often taking the values $\hbar=0.5,1$, or 2 .

In OpenFermion, the quadrature operators are represented by the QuadOperator class, and stored as a dictionary of tuples (as keys) and coefficients (as values), similar to the QubitOperator. For example, the multi-mode quadrature operator $q_{0} p_{1} q_{3}$ is represented internally as $((0$, ' $q$ '), ( 1 , ' $p$ '), (3, ' $q$ ')). Alternatively, QuadOperators also support string input — to initialize the latter operator using string representation, the user would enter the following: 
from openfermion.ops import QuadOperator

$\mathrm{O}=$ QuadOperator (' q0 p1 q3')

As with the BosonOperator, quadrature operators acting on different subsystems commute, so the internal representation orders the terms with lowest index to the left. We also define a "normal-ordering" for quadrature operators, to allow a unique representation. The normal-ordered function introduced earlier rearranges the terms via the commutation relation such that position operators $q_{i}$ always appear to the left of momentum operators $p_{i}$ :

normal_ordered (QuadOperator ('p0 q0'), hbar =2.)

Note that we now also pass the value $\hbar$ that appears in the commutation relation; if not provided, the default is $\hbar=1$.

Finally, OpenFermion provides functions for converting between the BosonOperator and QuadOperator:

from openfermion.transforms import get_quad_operator, get_boson_operator

$\mathrm{O}=$ QuadOperator ('q0 p1 q3')

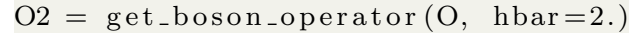

As before, we pass the value of $\hbar$ required depending on the convention chosen.

In addition to the functions and methods shown here, the BoseOperator and QuadOperator support additional operations (for example, sparse operator representations, symmetric ordering, and Weyl quantization of observables in the phase space). They also form the basis for models provided by OpenFermion, such as the Bose-Hubbard Hamiltonian.

\section{The MolecularData data structure}

While the FermionOperator, BosonOperator, and QubitOperator classes form the backbone of many of the internal computations for OpenFermion, the data that defines a particular physical problem is more conveniently stored within a separate well-defined object, namely, the MolecularData object. This defines the schema by which the intermediate quantities calculated for the electronic structure of a molecule are stored, such as the two-electron integrals, basis transformation from the original basis, energies from correlated methods, and meta data related to the computation. For an exhaustive list of the current information stored within this class, it is recommended to see the documentation, as quantities are added as needed.

Importantly, this information is stored to disk in an HDF5 format using the h5py package. This allows for seamless data access to the files for only the quantities of interest, without the need for loading the whole file or complicated interface structures. Internally this is performed through the use of getters and setters using Python decorators, but this is transparent to the user, needing only to get or set in the normal way, e.g.

two_body_integrals $=$ h2_molecule.two_body_integrals

where the data is read from disk on the access to two_body_integrals rather than when the object is instantiated in the first line. This controls the memory impact of larger objects such as the two-electron integrals. Compression functionality is also enabled through gzip to minimize the disk space requirements to store larger molecules.

Functionality is also built into MolecularData class to perform simple activate space approximations to the problem. An active space approximation isolates a subset of the total orbitals and treats the problem within that orbital set. This is done by modifying the one- and two-electron integrals as well as the count of active electrons and orbitals within the molecule. We assume a single reference for the active space definition and the occupied orbitals are integrated out according to the integrals while inactive virtual orbitals are removed. In OpenFermion this is as simple as taking a calculated molecule data structure, forming a list of the occupied spatial orbital indices (those which will be frozen in place) and active spatial orbital indices (those which may be freely occupied or unoccupied), and calling for some molecule larger than $\mathrm{H}_{2}$ in a minimal basis

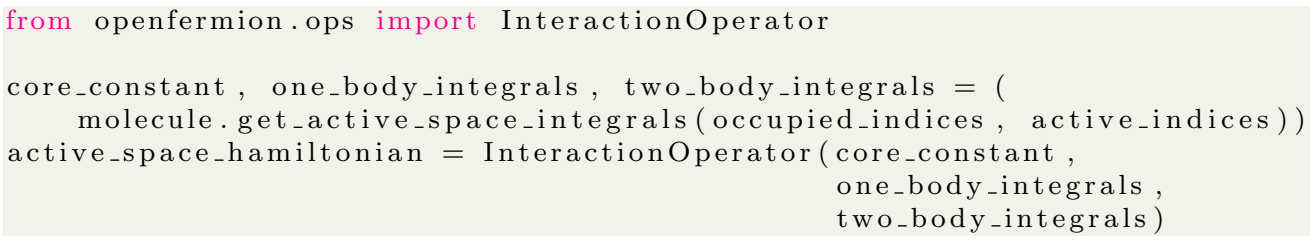

where active_space_hamiltonian can now be used to build quantum circuits for the reduced size problem. 


\section{E. The InteractionOperator data structure}

As OpenFermion deals primarily with the interactions of physical fermions, especially electrons, the Hamiltonian we have already introduced above

$$
H=h_{0}+\sum_{p q} h_{p q} a_{p}^{\dagger} a_{q}+\frac{1}{2} \sum_{p q r s} h_{p q r s} a_{p}^{\dagger} a_{q}^{\dagger} a_{r} a_{s}
$$

is ubiquitous throughout OpenFermion.

Note that even for fewer than $N=100$ qubits, the coefficients of the terms in the Hamiltonian of Eq. (18) can require a large amount of memory. Since common Gaussian basis functions lead to the full $\mathcal{O}\left(N^{4}\right)$ term scaling, instances with less than a hundred qubits can already have tens of millions to hundreds of millions of terms requiring on the order of ten gigabytes to store. Such large Hamiltonians can be expensive to generate (requiring nearly as many integrals as there are terms) so one would often like to be able to save these Hamiltonians. While good for general purpose symbolic manipulation, the FermionOperator data structure is not the most efficient way to store these Hamiltonians, or to manipulate them with efficient numerical linear algebra routines, due to the extra overhead of storing each of the associated operators.

Towards this end we introduce the InteractionOperator data structure. This structure has already been seen in passing in this document in the first example given. For example, with a MolecularData object, one may extract the Hamiltonian in the form of an InteractionOperator through

h2_hamiltonian $=$ h2_molecule.get_molecular_hamiltonian ()

where the Hamiltonian will be returned as an InteractionOperator. The InteractionOperator data structure is a class that stores two different matrices and a constant. In the notation of Eq. (18), the InteractionOperator stores the constant $h_{0}$, an $N$ by $N$ array representing $h_{p q}$ and an $N$ by $N$ by $N$ by $N$ array representing $h_{p q r s}$. Note that at present this is not a spatially optimal representation if the goal is specifically to store the integrals of molecular electronic structure systems, but it is a good compromise between space efficiency, simplicity, and ease of performing other numerical operations. The reason it is suboptimal is that there may exist symmetries within the integrals that are not currently utilized. For example, there is an eight-fold symmetry in the integrals for real basis functions, $h_{p q r s}=h_{s q r p}=h_{p r q s}=h_{s r q p}=h_{q p s r}=h_{r p s q}=h_{q s p r}=h_{r s p q}$. Note that for complex basis functions there is only a four-fold symmetry. While we provide methods to iterate over these unique elements, we do not exploit this symmetry in our current implementation for the reason that space efficiency of the InteractionOperator has not yet been a bottleneck in applications. This is consistent with our general design philosophy which is to maintain an active cycle of develop, test, profile, and refine. That is, rather than guess bottlenecks, we analyze and identify the most important ones for problems of interest, and focus time there.

\section{F. The InteractionRDM data structure}

As discussed in the previous section, since fermions are identical particles which interact pairwise, their energy can be determined entirely by reduced density matrices which are polynomial in size. In particular, these energies depend on the one-particle reduced density matrix (1-RDM), denoted here by ${ }^{(1)} D$ and the two-particle reduced density matrix $(2-\mathrm{RDM})$, denoted here by ${ }^{(2)} \mathrm{D}$. The 1-RDM and 2-RDM of a fermionic wavefunction $|\psi\rangle$ are defined through expectation values with one- and two-body local FermionOperators;

$$
{ }^{(1)} D_{p q}=\left\langle\psi\left|a_{p}^{\dagger} a_{q}\right| \psi\right\rangle \quad{ }^{(2)} D_{p q r s}=\left\langle\psi\left|a_{p}^{\dagger} a_{q}^{\dagger} a_{r} a_{s}\right| \psi\right\rangle \text {. }
$$

Thus, the 1-RDM is an $N$ by $N$ matrix and the 2-RDM is an $N$ by $N$ by $N$ by $N$ tensor. Note that the 1-RDM and 2-RDMs may (in general) represent a partial tomography of mixed states, in which case Eq. (19) should involve traces over that mixed state instead of expectation values with a pure state. If one has performed a computation at the CISD level of theory, it is possible to extract that density matrix from a molecule using the following command

cisd_two_rdm $=\mathrm{h} 22_{-}$molecule.get_molecular_rdm()

We can see that the energy of $|\psi\rangle$ with a Hamiltonian expressed in the form of Eq. (18) is given exactly as

$$
\langle E\rangle=h_{0}+\sum_{p, q}^{(1)} D_{p q} h_{p q}+\frac{1}{2} \sum_{p, q, r, s}{ }^{(2)} D_{p q r s} h_{p q r s}
$$

where $h_{0}, h_{p q}$ and $h_{p q r s}$ are the integrals stored by the InteractionOperator data structure. 
In OpenFermion, the InteractionRDM class provides an efficient numerical representation of these reduced density matrices. Both InteractionRDM and InteractionOperator inherit from a similar parent class, the PolynomialTensor, reflecting the close parallels between the implementation of these data structures. Due to this parallel, the exact same code which implements integral basis transformations on InteractionOperator also implements integral basis transformations on the InteractionRDM data structure. Despite their similarities, they represent conceptually distinct concepts, and in many cases should be treated in a fundamentally different way. For this reason the implementations are kept distinct.

\section{G. The QuadraticHamiltonian data structure}

The general electronic structure Hamiltonian Eq. (18) contains terms that act on up to 4 sites, or is quartic in the fermionic creation and annihilation operators. However, in many situations we may fruitfully approximate these Hamiltonians by replacing these quartic terms with terms that act on at most 2 fermionic sites, or quadratic terms, as in mean-field approximation theory. These Hamiltonians have a number of special properties one can exploit for efficient simulation and manipulation of the Hamiltonian, thus warranting a special data structure. We refer to Hamiltonians which only contain terms that are quadratic in the fermionic creation and annihilation operators as quadratic Hamiltonians, and include the general case of non-particle conserving terms as in a general Bogoliubov transformation. Eigenstates of quadratic Hamiltonians can be prepared efficiently on both a quantum and classical computer, making them amenable to initial guesses for many more challenging problems.

A general quadratic Hamiltonian takes the form

$$
\sum_{p, q}\left(M_{p q}-\mu \delta_{p q}\right) a_{p}^{\dagger} a_{q}+\frac{1}{2} \sum_{p, q}\left(\Delta_{p q} a_{p}^{\dagger} a_{q}^{\dagger}+\Delta_{p q}^{*} a_{q} a_{p}\right)+\text { constant }
$$

where $M$ is a Hermitian matrix, $\Delta$ is an antisymmetric matrix, $\delta_{p q}$ is the Kronecker delta symbol, and $\mu$ is a chemical potential term which we keep separate from $M$ so that we can use it to adjust the expectation of the total number of particles. In OpenFermion, quadratic Hamiltonians are conveniently represented and manipulated using the QuadraticHamiltonian class, which stores $M, \Delta, \mu$ and the constant from Eq. (21). It is specialized to exploit the properties unique to quadratic Hamiltonians. Examples showing the use of this class for simulation are provided later in this document.

\section{H. Some examples justifying data structure design choices}

Here we describe and show a few example illustrating that the above data structures are well designed for efficient calculations that one might do with OpenFermion. These examples are motivated by real use case examples encountered in the authors' own research. We do not provide code examples in all cases here but routines for these calculations exist within OpenFermion.

\section{FermionOperator example: computation of Trotter error operators}

Suppose that one has a Hamiltonian $H=\sum_{\ell=1}^{L} H_{\ell}$ where the $H_{\ell}$ are single-term FermionOperators. Suppose now that one decides to effect evolution under this Hamiltonian using the second-order Trotter formula, as investigated in works such as $[19,25,34-36]$. A single second-order Trotter step of this Hamiltonian effects evolution under $H+V$ where $V$ is the Trotter error operator which arises due to the fact that the $H_{\ell}$ do not all commute. In [35] it is shown that a perturbative approximation to the operator $V$ can be expressed as

$$
V^{(1)}=-\frac{1}{12} \sum_{\alpha \leq \beta} \sum_{\beta} \sum_{\gamma<\beta}\left[H_{\alpha}\left(1-\frac{\delta_{\alpha, \beta}}{2}\right),\left[H_{\beta}, H_{\gamma}\right]\right] .
$$

Because triangle inequality upper-bounds to this operator provide an estimate of the Trotter error which can be computed in polynomial time, symbolic computation of this operator is crucial for predicting how many Trotter steps one should take in a quantum simulation. However, when using conventional Gaussian basis functions, Hamiltonians of fermionic systems contain $\mathcal{O}\left(N^{4}\right)$ terms, suggesting that the number of terms in Eq. (22) could be as high as $\mathcal{O}\left(N^{10}\right)$. But in practice, numerics have shown that there is very significant cancellation in these commutators which leads to a number of nontrivial terms that is closer to $\mathcal{O}\left(N^{4}\right)$ after normal ordering [36]. If one were to use an array or 
linked list structure to store FermionOperators then one has two choices. Option (i) is that new terms from the sum are appended to the list and then combined after the sum is completed. Under this strategy the space complexity of the algorithm would scale as $\mathcal{O}\left(N^{10}\right)$ and one would likely run out of memory for medium sized systems. Option (ii) is that one normal orders the commutators before adding to the list and then loops through the array or list before adding each term. While this approach has average space complexity of $\mathcal{O}\left(N^{4}\right)$, the time complexity of this approach would then be $\mathcal{O}\left(N^{14}\right)$ as one would need to loop through $\mathcal{O}\left(N^{4}\right)$ entries in the list after computing each of the $\mathcal{O}\left(N^{10}\right)$ terms in the sum. Using our hash table implementation, the average space complexity is still $\mathcal{O}\left(N^{4}\right)$ but one does not need to loop through all entries at each iteration so the time complexity becomes $\mathcal{O}\left(N^{10}\right)$.

Though still quite expensive, one can use Monte Carlo based sampling or distribute this task on a cluster (it is embarrassingly parallel) to compute the Trotter error for medium sized systems. Using the Hamiltonian representations introduced in [25], Hamiltonians have only $\mathcal{O}\left(N^{2}\right)$ terms, which brings the number of terms in the sum down to $\mathcal{O}\left(N^{4}\right)$ in the worst case. In that case, computation of $V^{(1)}$ would have time complexity $\mathcal{O}\left(N^{4}\right)$ using the hash table implementation instead of $\mathcal{O}\left(N^{6}\right)$ complexity using either a linked-list or an array. The $\mathcal{O}\left(N^{4}\right)$ complexity enables us to compute $V^{(1)}$ for hundreds of qubits, well into the regime of instances that would be classically intractable to simulate. The task is even more efficient for Hubbard models which have $\mathcal{O}(N)$ terms.

\section{FermionOperator example: symbolic Fourier transformation}

A secondary goal for OpenFermion is that the library can be used to as a tool for the symbolic manipulation of fermionic Hamiltonians in order to analyze and develop new simulation algorithms and Hamiltonian representations. To give a concrete example of this, in the recent paper [25], authors were able to demonstrate Trotter steps of the electronic structure Hamiltonian with significantly reduced complexity: $\mathcal{O}(N)$ depth as opposed to $\mathcal{O}\left(N^{4}\right)$ depth. A critical component of that improvement was to represent the Hamiltonian using basis functions which are a discrete Fourier transform of the plane wave basis (the plane wave dual basis) [25]. The appendices of that paper begin by showing the Hamiltonian in the plane wave basis:

$$
H=\frac{1}{2} \sum_{p, \sigma} k_{p}^{2} c_{p, \sigma}^{\dagger} c_{p, \sigma}-\frac{4 \pi}{\Omega} \sum_{\substack{p \neq q \\ j, \sigma}}\left(\zeta_{j} \frac{e^{i k_{p-q} \cdot R_{j}}}{k_{q-p}^{2}}\right) c_{p, \sigma}^{\dagger} c_{q, \sigma}+\frac{2 \pi}{\Omega} \sum_{\substack{p, \sigma) \neq\left(q, \sigma^{\prime}\right) \\ \nu \neq 0}} \frac{c_{p, \sigma}^{\dagger} c_{q, \sigma^{\prime}}^{\dagger} c_{q+\nu, \sigma^{\prime}} c_{p-\nu, \sigma}}{k_{\nu}^{2}} .
$$

To obtain the Hamiltonian in the plane wave dual basis, one applies the Fourier transform of the mode operators,

$$
c_{\nu}^{\dagger}=\sqrt{\frac{1}{N}} \sum_{p} a_{p}^{\dagger} e^{-i k_{\nu} \cdot r_{p}} \quad c_{\nu}=\sqrt{\frac{1}{N}} \sum_{p} a_{p} e^{i k_{\nu} \cdot r_{p}} .
$$

Using OpenFermion one can easily generate the plane wave Hamiltonian (either manually or by using the plane wave module) and then apply the discrete Fourier transform of the mode operators (either manually or by using the discrete Fourier transform module) to verify the correct form of the plane wave dual Hamiltonian shown in [25],

$$
H=\frac{1}{2 N} \sum_{\nu, p, q, \sigma} k_{\nu}^{2} \cos \left[k_{\nu} \cdot r_{q-p}\right] a_{p, \sigma}^{\dagger} a_{q, \sigma}-\frac{4 \pi}{\Omega} \sum_{\substack{p, \sigma \\ j, \nu \neq 0}} \frac{\zeta_{j} e^{i k_{\nu} \cdot\left(R_{j}-r_{p}\right)}}{k_{\nu}^{2}} n_{p, \sigma}+\frac{2 \pi}{\Omega} \sum_{\substack{(p, \sigma) \neq\left(q, \sigma^{\prime}\right) \\ \nu \neq 0}} \frac{\cos \left[k_{\nu} \cdot r_{p-q}\right]}{k_{\nu}^{2}} n_{p, \sigma} n_{q, \sigma^{\prime}}
$$

While Eq. (25) turns out to have a very compact representation, it requires a careful derivation to show that application of Eq. (24) to Eq. (23) leads to Eq. (25). However, this task is trivial for OpenFermion since the Fourier transform can be applied symbolically and the output FermionOperator can be simplified automatically using a normal-ordering routine. This example demonstrates the utility of OpenFermion for verifying analytic calculations.

\section{InteractionOperator example: fast orbital basis transformations}

An example of an important numerical operation which is particularly efficient in this representation is a rotation of the molecular orbitals $\phi_{p}(r)$. This unitary basis transformation takes the form

$$
\tilde{\phi}_{p}(r)=\sum_{q=1}^{N} \phi_{q}(r) U_{p q} \quad U=e^{-\kappa} \quad \kappa=-\kappa^{\dagger}=\sum_{p, q} \kappa_{p q} a_{p}^{\dagger} a_{q}
$$


For $\kappa$ and $U$, the quantities $\kappa_{p q}$ and $U_{p q}$ respectively correspond to the matrix elements of these $N$ by $N$ matrices. We see then that the $\mathcal{O}\left(N^{2}\right)$ elements of the matrix $\kappa$ define a unitary transformation on all orbitals. Often, one would like to apply this transformation to Hamiltonian in the orbital basis defined by $\left\{\phi_{p}(r)\right\}$ in order to obtain a new Hamiltonian in the orbital basis defined by $\left\{\tilde{\phi}_{p}(r)\right\}$. Since computation of the integrals is extremely expensive, the goal is usually to apply this transformation directly to the Hamiltonian operator.

When specialized to real, orthogonal rotations $U$ (which is often the case in molecular electronic structure), the most straightforward expression of this integral transformation for the two-electron integrals is

$$
\tilde{h}_{p q r s}=\sum_{\mu, \nu, \lambda, \sigma} U_{p \mu} U_{q \nu} h_{\mu \nu \lambda \sigma} U_{r \lambda} U_{s \sigma} .
$$

Since there are $\mathcal{O}\left(N^{4}\right)$ integrals, the entire transformation of the Hamiltonian would take time $\mathcal{O}\left(N^{8}\right)$, which is extremely onerous. A more efficient approach is to rearrange this expression to obtain the following,

$$
\tilde{h}_{p q r s}=\sum_{\mu} U_{p \mu}\left[\sum_{\nu} U_{q \nu}\left[\sum_{\lambda} U_{r \lambda}\left[\sum_{\sigma} U_{s \sigma} h_{\mu \nu \lambda \sigma}\right]\right]\right]
$$

which can be evaluated for each term at cost $\mathcal{O}(N)$ since each summation can be carried out independently. This brings the total cost of the integral transformation to $\mathcal{O}\left(N^{5}\right)$. While such a transformation would be extremely tedious using the FermionOperator representation, this approach is implemented for InteractionOperators in OpenFermion using the einsum function for numerical linear algebra from numpy.

This functionality is readily accessible within OpenFermion for basis rotations. For example, the one may rotate the basis of the molecular hamiltonian of $\mathrm{H}_{2}$ to a new basis with some orthogonal matrix $U=\exp (-\kappa)$ of appropriate dimension as

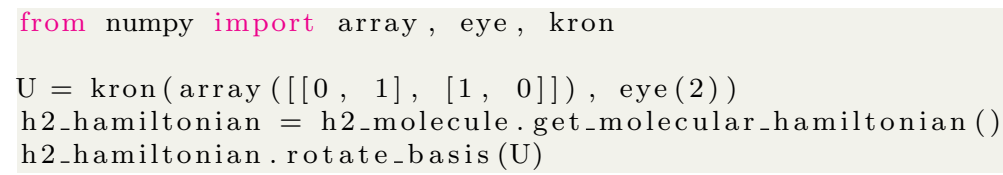

We now provide an example of where such fast basis transformations may be useful. Previous work has shown that the number of measurements required for a variational quantum algorithm to estimate the energy of a Hamiltonian such as Eq. (18) to precision $\epsilon$ scales as

$$
M=\mathcal{O}\left(\left[\frac{1}{\epsilon} \sum_{p, q, r, s}\left|h_{p q r s}\right|\right]^{2}\right) .
$$

Since the $h_{p q r s}$ are determined by the orbital basis, one can alter this bound by rotating the orbitals under angles organized into the $\kappa$ matrix of Eq. (26). Thus, one may want to perform an optimization over these angles in order to minimize $M$. This task would be quite unwieldy or perhaps nearly impossible using the FermionOperator data structure but is viable using the InteractionOperator data structure with fast integral transformations.

\section{QuadraticHamiltonian example: preparing fermionic Gaussian states}

As mentioned above, eigenstates of quadratic Hamiltonians, Eq. (21), can be prepared efficiently on a quantum computer, and OpenFermion includes functionality for compiling quantum circuits to prepare these states. Eigenstates of general quadratic Hamiltonians with both particle-conserving and non-particle conserving terms are also known as fermionic Gaussian states. A key step in the preparation of a fermionic Gaussian state is the computation of a basis transformation which puts the Hamiltonian of Eq. (21) into the form

$$
\sum_{p} \varepsilon_{p} b_{p}^{\dagger} b_{p}+\text { constant }
$$

where the $b_{p}^{\dagger}$ and $b_{p}$ are a new set of fermionic creation and annihilation operators that also obey the canonical fermionic anticommutation relations. In OpenFermion, this basis transformation is computed efficiently with matrix manipulations by exploiting the special properties of quadratic Hamiltonians that allow one to work with only the matrices $M$ and $\Delta$ from Eq. (21) stored by the QuadraticHamiltonian class, using simple numerical linear algebra 
routines included in SciPy. The following code constructs a mean-field Hamiltonian of the $d$-wave model of superconductivity and then obtains a circuit that prepares its ground state (along with a description of the starting state to which the circuit should be applied):

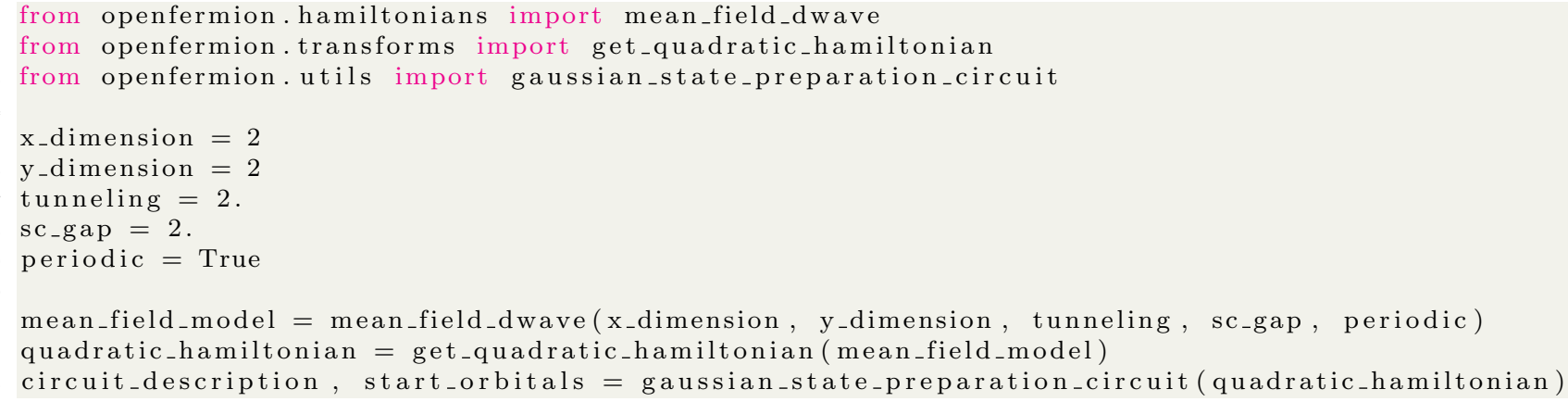

The circuit description follows the procedure explained in [10]. One can also obtain the ground energy and ground state numerically with the following code:

from openfermion. utils import jw_get_gaussian_state

ground_energy, ground_state = jw_get_gaussian_state(quadratic_hamiltonian)

\section{MODELS AND UTILITIES}

OpenFermion has a number of capabilities that assist with the creation and manipulation of fermionic and related Hamiltonians. While they are too numerous and growing too quickly to list in their entirety, here we will briefly describe a few of these examples in an attempt to ground what one would expect to find within OpenFermion.

One broad class of functions found within OpenFermion is the generation of fermionic and related Hamiltonians. While the MolecularData structure discussed above is one example for molecular systems, we also include utilities for a number of model systems of interest as well. For example, there are currently supported routines for generating Hamiltonians of the Hubbard model, the homogeneous electron gas (jellium), general plane wave discretizations, and $d$-wave models of superconductivity.

To give a concrete example, if one wished to study the homogeneous electron gas in $2 \mathrm{D}$, which is of interest when studying the fractional quantum hall effect, then one could use OpenFermion to initialize the model as follows

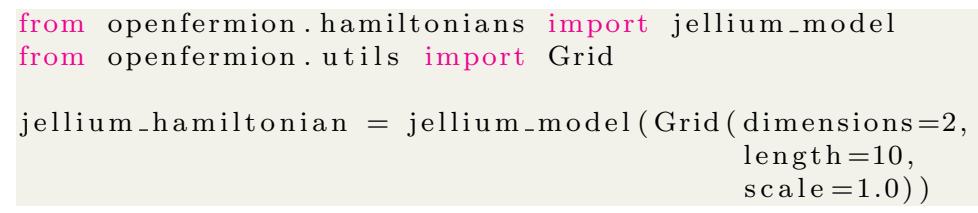

where jellium_hamiltonian will be a fermionic operator representing the spinful homogeneous electron gas discretized into a $10 \times 10$ grid of plane waves in two dimensions. One is then free to transform this Hamiltonian into qubit operators and use it in the algorithm of choice.

Similarly, one may use the utilities provided to prepare a Fermi-Hubbard model for study. For example

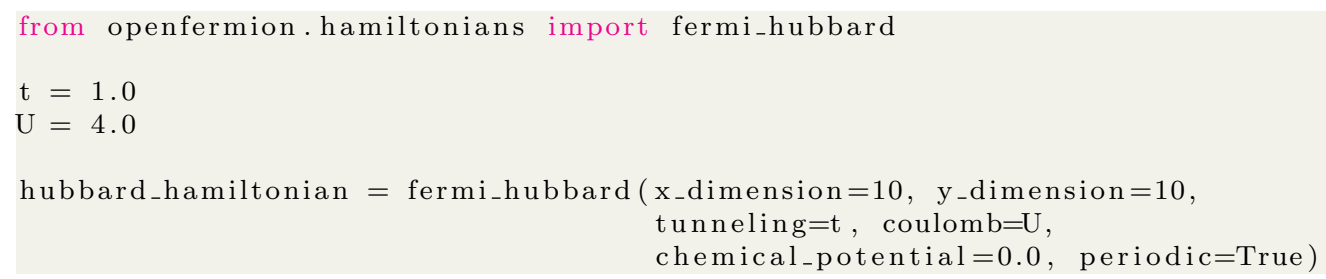

creates a Fermi-Hubbard Hamiltonian on a square lattice in $2 \mathrm{D}$ that is $10 \times 10$ sites with periodic boundary conditions.

Besides Hamiltonian generation, OpenFermion also includes methods for outputting Trotter-Suzuki decompositions of arbitrary operators, providing the corresponding quantum circuit in QASM format [87]. These methods were included in order to simplify porting the resulting quantum circuits to other simulation packages, such as LIQUi| $>$ [75], qTorch [79], Project-Q [70], and qHipster [88]. For instance, the function pauli_exp_to_qasm takes a list of QubitOperators and an optional evolution time as input, and outputs the QASM specification as a string. As an example, 


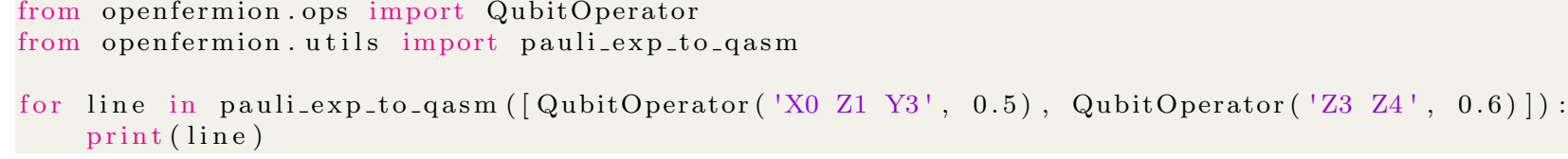

outputs a QASM specification for a circuit corresponding to $U=e^{-i 0.5 X_{0} Z_{1} Y 3} e^{-i 0.6 Z_{3} Z_{4}}$, which in this case is given by

$\mathrm{H} 0$

$\mathrm{Rx} \quad 1.5707963267948966 \quad 3$

CNOT 01

CNOT 1 1 3

$\mathrm{Rz} 0.53$

CNOT 13

CNOT 01

$\mathrm{H} 0$

$\mathrm{Rx}-1.5707963267948966 \quad 3$

CNOT 34

$\mathrm{Rz} \quad 0.6 \quad 4$

CNOT $3 \quad 4$

OpenFermion additionally supports a number of other tools including the ability to evaluate the Trotter error operator and construct circuits for preparing arbitrary Slater determinants on a quantum computer using the linear depth procedure described in [10]. Some support for numerical simulation is included for testing purposes, but the heavy lifting in this area is delegated to plugins specialized for these applications. In the future, we imagine these utilities will expand to include more Hamiltonians and specializations that add in the creation of simulation circuits for fermionic systems.

\section{OPEN SOURCE MANAGEMENT AND PROJECT PHILOSOPHY}

OpenFermion is designed to be a tool for both its developers and the community at large. By maintaining an opensource and framework independent library, we believe it provides a useful tool for developers in industry, academia, and research institutions alike. Moreover, it is our hope that these developers will, in turn, contribute code they found to be useful in interacting with OpenFermion to the benefit of the field as a whole. Here we outline some of our philosophy, as well as the ways in which we ensure that OpenFermion remains a high quality package, even as many different developers from different institutions contribute.

\section{A. Style and testing}

The OpenFermion code is written primarily in Python with optional $\mathrm{C}++$ backends being introduced as higher performance is desired. Stylistically, this code follows the PEP8 guidelines for Python and demands descriptive names as well as extensive documentation for all functions. This enhances readability and allows developers to more reliably build off of contributed code.

At present, the source code is managed through GitHub where the standard pull request system is used for making contributions. When a pull request is made, it must be reviewed by at least one member of the OpenFermion team who has experience with the library and is able to comment on both the style and integration prospects with the library. As contributors become more familiar with the code, they may become approved reviewers themselves to enhance their contribution to the process. All contributors are welcome to assist with reviews but reviews from new contributors will not automatically enable merging into the master branch.

Tests in the code are written in the python unittest framework and all code is required to be both Python 2 and Python 3 compliant so that it continues to be as useful as possible for all users in the future. The tests are run automatically when a pull request is made through the Travis continuous integration (CI) framework, to minimize the chance that code that will break crucial functionality is accidentally merged into the code base, and ensure a smooth development and execution experience.

\section{B. Distribution}

Several options for code distribution are available to obtain OpenFermion. The code may be pulled and used directly from the GitHub repository if desired (which one can find at www.openfermion.org), and the requirements 
may be manually fulfilled by the user. This option offers maximum control and access to bleeding edge code and developments, but minimal convenience. A full service option is offered through the Python Package Index (PyPI) so that installation for most users can be as simple as

python $-\mathrm{m}$ pip install openfermion

A middle ground between these options which is popular with many of the lead developers of this project is to pull the latest code directly from GitHub but to install with pip using the development install command in the OpenFermion directory:

python $-\mathrm{m}$ pip install -e

In the future, a version of the code may be supported for other distribution platforms as well.

The OpenFermion plugins (and the packages on which they rely) need to be installed independently using a similar procedure. One can install OpenFermion-Psi4 using pip under the name "openfermionpsi4", OpenFermion-Cirq under the name "openfermioncirq" and OpenFermion-PySCF under the name "openfermionpyscf". These plugins can also be installed directly from GitHub (we link to repositories from the main OpenFermion page).

In addition to the traditional installation models of either installing from the PyPI registry, or downloading the source from GitHub and installing, the project also supports a Docker container. Docker containers offer a compact virtualization environment that is portable between all systems where Docker is supported. This is a convenient option for both first time users, and those who want to deploy OpenFermion to non-standard architectures (or on Windows). Moreover, it offers easy access to some of the electronic structure packages that our plugins are inter-operable with, which allows users convenient access to the full tool chain. At present, the Dockerfile is hosted on the repository, which can be used to build a Docker image as detailed in full in the Readme of the Docker folder.

\section{CLOSING REMARKS}

The rapid development of quantum hardware represents an impetus for the equally rapid development of quantum applications. Development of these applications represents a unique challenge, often requiring the expertise or domain knowledge from both the application area and quantum algorithms. OpenFermion is a bridge between the world of quantum computing and materials simulation, which we believe will act as a catalyst for development in this critical area. Only with such software tools can we start to explore the explicit costs and advantages of new algorithms, and push forward with practical advancements. It is our hope that OpenFermion not only leads to developments in the field of quantum computation for quantum chemistry and materials, but also sparks the development of similar packages for other application areas.

\section{ACKNOWLEDGEMENTS}

The authors thank Hartmut Neven for encouraging the initiation of this project at Google as well as Alán AspuruGuzik, Carlo Beenakker, Yaoyun Shi, Matthias Troyer, Stephanie Wehner and James Whitfield for supporting graduate student and postdoc developers who contributed code to OpenFermion. I. D. K. acknowledges partial support from the National Sciences and Engineering Research Council of Canada. K. J. S. acknowledges support from NSF Grant No. 1717523 . T. H. and D. S. S. have been supported by the Swiss National Science Foundation through the National Competence Center in Research QSIT. S. S. is supported by the DOE Computational Science Graduate Fellowship under grant number DE-FG02-97ER25308. MS is supported by the Netherlands Organization for Scientific Research (NWO/OCW) and an ERC Synergy Grant.

[1] P. W. Shor, SIAM Journal on Computing 26, 1484 (1997).

[2] A. W. Harrow, A. Hassidim, and S. Lloyd, Phys. Rev. Lett. 103, 150502 (2009).

[3] R. P. Feynman, International Journal of Theoretical Physics 21, 467 (1982).

[4] S. Lloyd, Science 273, 1073 (1996).

[5] D. S. Abrams and S. Lloyd, Physical Review Letters 79, 4 (1997).

[6] D. S. Abrams and S. Lloyd, Physical Review Letters 83, 5162 (1999).

[7] G. Ortiz, J. Gubernatis, E. Knill, and R. Laflamme, Physical Review A 64, 22319 (2001).

[8] R. D. Somma, G. Ortiz, J. E. Gubernatis, E. Knill, and R. Laflamme, Physical Review A 65, 17 (2002).

[9] A. Aspuru-Guzik, A. D. Dutoi, P. J. Love, and M. Head-Gordon, Science 309, 1704 (2005). 
[10] Z. Jiang, K. J. Sung, K. Kechedzhi, V. N. Smelyanskiy, and S. Boixo, Physical Review Applied 9, 044036 (2018).

[11] D. Wecker, M. B. Hastings, N. Wiebe, B. K. Clark, C. Nayak, and M. Troyer, Physical Review A 92, 62318 (2015).

[12] I. Kivlichan, N. Wiebe, C. Gidney, J. McClean, W. Sun, V. Denchev, A. Fowler, A. Aspuru-Guzik, and R. Babbush, Unpublished (2018).

[13] L. García-Álvarez, I. L. Egusquiza, L. Lamata, A. del Campo, J. Sonner, and E. Solano, Physical Review Letters 119, 040501 (2017).

[14] R. Babbush, D. Berry, and H. Neven, arXiv:1806.02793 (2018).

[15] I. Kassal, S. P. Jordan, P. J. Love, M. Mohseni, and A. Aspuru-Guzik, Proceedings of the National Academy of Sciences 105, 18681 (2008).

[16] N. J. Ward, I. Kassal, and A. Aspuru-Guzik, Journal Of Chemical Physics 130, 194105 (2008).

[17] J. D. Whitfield, J. Biamonte, and A. Aspuru-Guzik, Molecular Physics 109, 735 (2011).

[18] B. Toloui and P. J. Love, arXiv:1312.2579 (2013).

[19] M. B. Hastings, D. Wecker, B. Bauer, and M. Troyer, Quantum Information \& Computation 15, 1 (2015).

[20] R. Babbush, D. W. Berry, Y. R. Sanders, I. D. Kivlichan, A. Scherer, A. Y. Wei, P. J. Love, and A. Aspuru-Guzik, Quantum Science and Technology 3, 015006 (2018).

[21] R. Babbush, D. W. Berry, I. D. Kivlichan, A. Y. Wei, P. J. Love, and A. Aspuru-Guzik, New Journal of Physics 18,33032 (2016).

[22] R. Babbush, P. J. Love, and A. Aspuru-Guzik, Scientific Reports 4 (2014), 10.1038/srep06603.

[23] I. D. Kivlichan, N. Wiebe, R. Babbush, and A. Aspuru-Guzik, Journal of Physics A: Mathematical and Theoretical 50, 305301 (2017).

[24] K. Sugisaki, S. Yamamoto, S. Nakazawa, K. Toyota, K. Sato, D. Shiomi, and T. Takui, The Journal of Physical Chemistry A 120, 6459 (2016).

[25] R. Babbush, N. Wiebe, J. McClean, J. McClain, H. Neven, and G. K.-L. Chan, Physical Review X 8, 011044 (2018).

[26] F. Motzoi, M. P. Kaicher, and F. K. Wilhelm, Physical Review Letters 119, 160503 (2017).

[27] I. D. Kivlichan, J. McClean, N. Wiebe, C. Gidney, A. Aspuru-Guzik, G. K.-L. Chan, and R. Babbush, Physical Review Letters 120, 110501 (2018).

[28] D. W. Berry, M. Kieferová, A. Scherer, Y. R. Sanders, G. H. Low, N. Wiebe, C. Gidney, and R. Babbush, npj Quantum Information 4, 22 (2018).

[29] R. Babbush, D. W. Berry, J. R. McClean, and H. Neven, arXiv:1807.09802 (2018).

[30] D. Berry, C. Gidney, M. Motta, J. McClean, and R. Babbush, arXiv:1902.02134 (2019).

[31] L. Veis and J. Pittner, Journal of Chemical Physics 133, 194106 (2010).

[32] L. Veis and J. Pittner, Journal of Chemical Physics 140, 1 (2014).

[33] J. R. McClean, R. Babbush, P. J. Love, and A. Aspuru-Guzik, The Journal of Physical Chemistry Letters 5, 4368 (2014).

[34] D. Wecker, B. Bauer, B. K. Clark, M. B. Hastings, and M. Troyer, Physical Review A 90, 1 (2014).

[35] D. Poulin, M. B. Hastings, D. Wecker, N. Wiebe, A. C. Doherty, and M. Troyer, Quantum Information \& Computation 15, 361 (2015).

[36] R. Babbush, J. McClean, D. Wecker, A. Aspuru-Guzik, and N. Wiebe, Physical Review A 91, 22311 (2015).

[37] N. Rubin, R. Babbush, and J. McClean, New Journal of Physics 20, 053020 (2018).

[38] J. T. Seeley, M. J. Richard, and P. J. Love, Journal of Chemical Physics 137, 224109 (2012).

[39] J. D. Whitfield, Journal of Chemical Physics 139, 21105 (2013).

[40] A. Tranter, S. Sofia, J. Seeley, M. Kaicher, J. McClean, R. Babbush, P. V. Coveney, F. Mintert, F. Wilhelm, and P. J. Love, International Journal of Quantum Chemistry 115, 1431 (2015).

[41] N. Moll, A. Fuhrer, P. Staar, and I. Tavernelli, Journal of Physics A: Mathematical and Theoretical 49, 295301 (2016).

[42] J. D. Whitfield, V. Havlíček, and M. Troyer, Physical Review A 94, 030301(R) (2016).

[43] P. K. Barkoutsos, N. Moll, P. W. J. Staar, P. Mueller, A. Fuhrer, S. Filipp, M. Troyer, and I. Tavernelli, arXiv:1706.03637 (2017).

[44] V. Havlíček, M. Troyer, and J. D. Whitfield, Physical Review A 95, 32332 (2017).

[45] S. Bravyi, J. M. Gambetta, A. Mezzacapo, and K. Temme, arXiv:1701.08213 (2017).

[46] G. Zhu, Y. Subasi, J. D. Whitfield, and M. Hafezi, arXiv:1707.04760 (2017).

[47] K. Setia and J. D. Whitfield, The Journal of Chemical Physics 148, 164104 (2018).

[48] M. Steudtner and S. Wehner, New Journal of Physics 20, 063010 (2018).

[49] M. Motta, E. Ye, J. R. McClean, Z. Li, A. J. Minnich, R. Babbush, and G. K.-L. Chan, arXiv:1808.02625 (2018).

[50] Z. Jiang, J. McClean, R. Babbush, and H. Neven, arXiv:1812.08190 (2018).

[51] N. Cody Jones, J. D. Whitfield, P. L. McMahon, M.-H. Yung, R. V. Meter, A. Aspuru-Guzik, and Y. Yamamoto, New Journal of Physics 14, 115023 (2012).

[52] M. Reiher, N. Wiebe, K. M. Svore, D. Wecker, and M. Troyer, Proceedings of the National Academy of Sciences 114, 7555 (2017).

[53] R. Babbush, C. Gidney, D. Berry, N. Wiebe, J. McClean, A. Paler, A. Fowler, and H. Neven, Physical Review X 8, 041015 (2018).

[54] B. P. Lanyon, J. D. Whitfield, G. G. Gillett, M. E. Goggin, M. P. Almeida, I. Kassal, J. D. Biamonte, M. Mohseni, B. J. Powell, M. Barbieri, A. Aspuru-Guzik, and a. G. White, Nature Chemistry 2, 106 (2010).

[55] Z. Li, M.-H. Yung, H. Chen, D. Lu, J. D. Whitfield, X. Peng, A. Aspuru-Guzik, and J. Du, Scientific Reports 1, 1 (2011). 
[56] Y. Wang, F. Dolde, J. Biamonte, R. Babbush, V. Bergholm, S. Yang, I. Jakobi, P. Neumann, A. Aspuru-Guzik, J. D. Whitfield, and J. Wrachtrup, ACS Nano 9, 7769 (2015).

[57] R. Santagati, J. Wang, A. Gentile, S. Paesani, N. Wiebe, J. McClean, S. Short, P. Shadbolt, D. Bonneau, J. Silverstone, D. Tew, X. Zhou, J. OBrien, and M. Thompson, arXiv:1611.03511 (2016).

[58] A. Peruzzo, J. McClean, P. Shadbolt, M.-H. Yung, X.-Q. Zhou, P. J. Love, A. Aspuru-Guzik, and J. L. O'Brien, Nature Communications 5, 1 (2014).

[59] M.-H. Yung, J. Casanova, A. Mezzacapo, J. McClean, L. Lamata, A. Aspuru-Guzik, and E. Solano, Scientific Reports 4, 9 (2014).

[60] J. R. McClean, J. Romero, R. Babbush, and A. Aspuru-Guzik, New Journal of Physics 18, 23023 (2016).

[61] Y. Shen, X. Zhang, S. Zhang, J.-N. Zhang, M.-H. Yung, and K. Kim, Physical Review A 95, 020501(R) (2017).

[62] D. Wecker, M. B. Hastings, and M. Troyer, Physical Review A 92, 42303 (2015).

[63] N. P. D. Sawaya, M. Smelyanskiy, J. R. McClean, and A. Aspuru-Guzik, Journal of Chemical Theory and Computation 12, 3097 (2016)

[64] J. R. McClean, M. E. Kimchi-Schwartz, J. Carter, and W. A. de Jong, Phys. Rev. A 95, 042308 (2017).

[65] P. J. J. O'Malley, R. Babbush, I. D. Kivlichan, J. Romero, J. R. McClean, R. Barends, J. Kelly, P. Roushan, A. Tranter, N. Ding, B. Campbell, Y. Chen, Z. Chen, B. Chiaro, A. Dunsworth, A. G. Fowler, E. Jeffrey, A. Megrant, J. Y. Mutus, C. Neill, C. Quintana, D. Sank, A. Vainsencher, J. Wenner, T. C. White, P. V. Coveney, P. J. Love, H. Neven, A. AspuruGuzik, and J. M. Martinis, Physical Review X 6, 31007 (2016).

[66] J. I. Colless, V. V. Ramasesh, D. Dahlen, M. S. Blok, J. R. McClean, J. Carter, W. A. de Jong, and I. Siddiqi, arXiv:1707.06408 (2017).

[67] A. Kandala, A. Mezzacapo, K. Temme, M. Takita, J. M. Chow, and J. M. Gambetta, Nature 549, 242 (2017).

[68] J. Romero, R. Babbush, J. McClean, C. Hempel, P. Love, and A. Aspuru-Guzik, Quantum Science and Technology 4, 14008 (2017).

[69] C. Hempel, C. Maier, J. Romero, J. McClean, T. Monz, H. Shen, P. Jurcevic, B. Lanyon, P. Love, R. Babbush, A. AspuruGuzik, R. Blatt, and C. Roos, Physical Review X 8, 031022 (2018).

[70] D. S. Steiger, T. Häner, and M. Troyer, arXiv:1612.08091 (2016).

[71] A. W. Cross, L. S. Bishop, J. A. Smolin, and J. M. Gambetta, arXiv:1707.03429 (2017).

[72] R. S. Smith, M. J. Curtis, and W. J. Zeng, "A practical quantum instruction set architecture," (2016).

[73] A. S. Green, P. L. Lumsdaine, N. J. Ross, P. Selinger, and B. Valiron, in Proceedings of the 34th ACM SIGPLAN Conference on Programming Language Design and Implementation, PLDI '13 (ACM, New York, NY, USA, 2013) pp. 333-342.

[74] P. Selinger, in Functional and Logic Programming (Springer Berlin Heidelberg, 2004) pp. 1-6.

[75] D. Wecker and K. M. Svore, arXiv:1402.4467 (2014).

[76] B. Valiron, N. J. Ross, P. Selinger, D. S. Alexander, and J. M. Smith, Commun. ACM 58, 52 (2015).

[77] J. Heckey, S. Patil, A. JavadiAbhari, A. Holmes, D. Kudrow, K. R. Brown, D. Franklin, F. T. Chong, and M. Martonosi, in ACM SIGARCH Computer Architecture News, Vol. 43 (ACM, 2015) pp. 445-456.

[78] A. JavadiAbhari, S. Patil, D. Kudrow, J. Heckey, A. Lvov, F. T. Chong, and M. Martonosi, in Proceedings of the 11th ACM Conference on Computing Frontiers (ACM, 2014) p. 1.

[79] E. S. Fried, N. P. Sawaya, Y. Cao, I. D. Kivlichan, J. Romero, and A. Aspuru-Guzik, arXiv:1709.03636 (2017).

[80] N. Killoran, J. Izaac, N. Quesada, V. Bergholm, M. Amy, and C. Weedbrook, arXiv preprint arXiv:1804.03159 (2018).

[81] T. Helgaker, P. Jorgensen, and J. Olsen, Molecular Electronic Structure Theory (Wiley, 2002).

[82] K. L. Schuchardt, B. T. Didier, T. Elsethagen, L. Sun, V. Gurumoorthi, J. Chase, J. Li, and T. L. Windus, Journal of Chemical Information and Modeling 47, 1045 (2007), pMID: 17428029, http://dx.doi.org/10.1021/ci600510j.

[83] R. M. Parrish, L. A. Burns, D. G. A. Smith, A. C. Simmonett, A. E. DePrince, E. G. Hohenstein, U. Bozkaya, A. Y. Sokolov, R. Di Remigio, R. M. Richard, J. F. Gonthier, A. M. James, H. R. McAlexander, A. Kumar, M. Saitow, X. Wang, B. P. Pritchard, P. Verma, H. F. Schaefer, K. Patkowski, R. A. King, E. F. Valeev, F. A. Evangelista, J. M. Turney, T. D. Crawford, and C. D. Sherrill, Journal of Chemical Theory and Computation 13, 3185 (2017), pMID: 28489372, http://dx.doi.org/10.1021/acs.jctc.7b00174.

[84] Q. Sun, T. C. Berkelbach, N. S. Blunt, G. H. Booth, S. Guo, Z. Li, J. Liu, J. McClain, S. Sharma, S. Wouters, et al., e-print arXiv:1701.08223 (2017).

[85] P. Jordan and E. Wigner, Zeitschrift für Physik 47, 631 (1928).

[86] S. Bravyi and A. Kitaev, Annals of Physics 298, 210 (2002).

[87] A. W. Cross, L. S. Bishop, J. A. Smolin, and J. M. Gambetta, arXiv:1707.03429 (2017).

[88] M. Smelyanskiy, N. P. D. Sawaya, and A. Aspuru-Guzik, "qHiPSTER: The Quantum High Performance Software Testing Environment," (2016), arXiv:1601.07195. 